\title{
Philosophiques
}

\section{Identité et égalité : le criticisme de Poincaré}

\section{Igor Ly}

Volume 31, numéro 1, printemps 2004

Poincaré et la théorie de la connaissance

URI : https://id.erudit.org/iderudit/008939ar

DOI : https://doi.org/10.7202/008939ar

Aller au sommaire du numéro

Éditeur(s)

Société de philosophie du Québec

ISSN

0316-2923 (imprimé)

1492-1391 (numérique)

Découvrir la revue

Citer cet article

Ly, I. (2004). Identité et égalité : le criticisme de Poincaré. Philosophiques, 31(1),

179-212. https://doi.org/10.7202/008939ar

\section{Résumé de l'article}

Ce travail a pour objet d'examiner comment sont articulés deux thèmes centraux de l'oeuvre philosophique de Poincaré : la question de la nature mathématique de la physique et la critique des questionnements

" métaphysiques » relatifs aux théories scientifiques. En s'appuyant sur la dimension linguistique du traitement de ces thèmes par Poincaré et en analysant certains aspects de ses réflexions sur le continu et la mesure des grandeurs physiques, notre examen sera poursuivi en suivant le fil conducteur de la distinction entre les notions d'identité numérique et d'égalité, dont nous tâcherons de montrer qu'elle sous-tend tout à la fois la caractérisation par Poincaré de la métaphysique et ses considérations sur la géométrie et le continu. 


\title{
Identité et égalité
}

\section{Le criticisme de Poincaré'}

\author{
IGOR LY \\ LPHS - Archives Poincaré (Université Nancy 2) \\ Université d'Angers \\ lyigor@club-internet.fr
}

\begin{abstract}
RÉSUMÉ. - Ce travail a pour objet d'examiner comment sont articulés deux thèmes centraux de l'œuvre philosophique de Poincaré: la question de la nature mathématique de la physique et la critique des questionnements «métaphysiques » relatifs aux théories scientifiques. En s'appuyant sur la dimension linguistique du traitement de ces thèmes par Poincaré et en analysant certains aspects de ses réflexions sur le continu et la mesure des grandeurs physiques, notre examen sera poursuivi en suivant le fil conducteur de la distinction entre les notions d'identité numérique et d'égalité, dont nous tâcherons de montrer qu'elle sous-tend tout à la fois la caractérisation par Poincaré de la métaphysique et ses considérations sur la géométrie et le continu.
\end{abstract}

\begin{abstract}
The purpose of this study is to examine the link between two main topics of Poincaré's philosophical work: the question of the mathematical nature of physics and the criticism of "metaphysical" questions about physical theories. By focusing on the linguistic dimension of Poincaré's treatment of these subjects and by analysing some aspects of his reflections about continuum and physical measurement, our inquiry will be pursued following the vital lead of the distinction between numerical identity and equality, which appears to underlie both Poincaré's characterisation of metaphysics and his reflections about geometry and the continuum.
\end{abstract}

La philosophie est écrite dans cet immense livre qui se tient toujours devant nos yeux, je veux dire l'Univers, mais on ne peut le comprendre si on ne s'applique d'abord à en comprendre la langue et à connaître les caractères avec lesquels il est écrit. Il est écrit dans la langue mathématique et ses caractères sont des triangles, des cercles et autres figures géométriques, sans le moyen desquels il est humainement impossible d'en comprendre un mot.

$$
\text { Galilée, Il Saggiatore, p. } 141 .
$$

[La science mathématique] n'a pas uniquement pour objet de contempler éternellement son propre nombril; elle touche à la nature et un jour ou l'autre elle prendra contact avec elle; ce jour-là, il faudra secouer les définitions purement verbales et ne plus se payer de mots.

Poincaré, Science et méthode, p. 158

1. Cet article est une version remaniée et développée d'un exposé donné le 18 décembre 2002 à l'Institut Henri Poincaré (Paris) dans le cadre de la journée «Il y a 100 ans: La Science et l'hypothèse", organisée par les Archives Poincaré sous le patronage de la Société mathématique de France. 
L'usage important que fait Poincaré de notions introduites par Kant («jugements synthétiques a priori », "catégories", etc.) rend manifeste l'influence de la philosophie critique sur la sienne. Entre autres et malgré les différences importantes qui séparent leurs traitements dans les deux œuvres, un thème et une question - ainsi que l'articulation étroite de leurs instructions respectives - leur sont communs. Le thème est celui de la recherche d'une ligne de démarcation entre la science et la métaphysique; la question est celle de la nature mathématique de la physique moderne ${ }^{2}$. C'est en référence à cette communauté thématique et problématique que nous parlerons ici d'un "criticisme" de Poincaré.

Sans poursuivre davantage la comparaison entre les philosophies de Kant et de Poincaré, qui appellerait des analyses et des développements dépassant largement le cadre de cette étude, prenons néanmoins pour point de départ de ce travail un texte tiré de la Critique de la raison pure, qui introduit à la première partie du titre choisi ici et qui rejoint l'idée que nous allons développer:

Quand un objet se présente à nous plusieurs fois, mais chaque fois avec les mêmes déterminations intérieures (qualitas et quantitas), il est, si on le considère comme un objet de l'entendement pur, le même, toujours le même, non pas plusieurs, mais une seule chose (numerica identitas); si au contraire il est phénomène, il ne s'agit plus de comparer des concepts, mais quelque identique que tout puisse être à ce point de vue, la diversité des lieux qu'occupe ce phénomène dans un même temps est un principe suffisant de la diversité numérique de l'objet (des sens) ${ }^{3}$.

Le but de la présente étude est de montrer que l'idée exprimée ici selon laquelle les phénomènes physiques ne sauraient être compris en termes d'identité, contrairement à la façon dont sont pensés les objets dans l'usage pur de l'entendement (tel qu'il se manifeste dans la métaphysique dogmatique) - est présente, mutatis mutandis, dans la philosophie de Poincaré et constitue un des éléments centraux des considérations qu'il déploie au sujet du thème et de la question évoqués plus haut. Nous verrons que l'impossibilité d'appliquer le principe d'identité aux phénomènes physiques ne peut être formulée, chez Poincaré, comme reposant sur la diversité des lieux et des temps. La considération de l'espace n'en est pas moins indispensable, mais sous une forme différente: celle de l'implication fondamentale de la géométrie dans l'opération de mesure. Précisons que la présente étude se concentre sur un seul aspect de cette implication: la question de la continuité mathématique.

2. Notons que cette double thématique est commune à de nombreux savants philosophes du XIX ${ }^{e}$ siècle, ce qui peut s'expliquer par les développements de la physique mathématique au cours de ce siècle. La radicalité du traitement que lui applique Poincaré et son tour linguistique en font, à notre sens, l'intérêt et l'originalité.

3. Kant, Critique de la raison pure, "De l'amphibologie des concepts de la réflexion, résultant de la confusion de l'usage empirique de l'entendement avec son usage transcendantal », Ak III, 216, 217. 
Outre l'inspiration thématique et problématique kantienne, les considérations philosophiques de Poincaré ici examinées peuvent être rapprochées à certains égards de celles des Remarques sur les fondements des mathématiques de Wittgenstein, notamment sur l'idée - centrale dans les critiques du logicisme développées par les deux auteurs - selon laquelle la grammaire profonde d'une expression ne coïncide pas avec sa grammaire de surface, dont nous allons voir qu'elle a une grande importance dans la distinction, affirmée par Poincaré, entre physique et métaphysique.

Dans cette perspective, une des thèses qui résulteront de notre analyse est la suivante: un énoncé de la forme $x=y$ ne saurait, de façon générale, être interprété, à la manière frégéenne, comme exprimant une identité (au sens où une telle proposition signifierait que les signes $x$ et $y$ renvoient à la même chose). À cet égard, on peut considérer que la philosophie de Poincaré anticipe et oppose une objection de fond aux travaux qui accordent une place importante à l'outil logique en philosophie des mathématiques en adoptant notamment cette interprétation du signe $=^{4}$. Nous tâcherons ainsi de suggérer que les considérations de Poincaré sur la métaphysique sont solidaires d'un aspect important de son opposition au logicisme considéré en un sens large, comprenant notamment le recours à l'outil logique en vue d'une clarification philosophique du discours scientifique ${ }^{5}$.

4. Citons par exemple:

- Quine, Le Mot et la chose, p. 171: «Le signe “=” de l'identité est un terme relationnel. [...] Comme tout terme relationnel, il relie des termes singuliers pour former une phrase. La phrase ainsi formée est vraie si et seulement si ses composants renvoient au même objet. »

- Benacerraf, "What Numbers could not be", p. 286: "If an expression of the form " $x=y "$ is to have a sense, it can be only in contexts where it is clear that both $x$ and $y$ are of some kind or category $\mathrm{C}$, and that it is the conditions which individuate things as the same $\mathrm{C}$ which are operative and determine its true value."

- Putnam, Philosophie de la logique, p. 27: "“=” (lire "est identique à", "est égal à") indique l'identité; ainsi, " $x=y$ " signifie " $x$ est identique à $y$ ", " $x$ et $y$ sont une seule et même entité”."

Précisons que ces textes sont extraits d'ouvrages ou d'articles dans lesquels il est fait une application de ces définitions au sein de considérations sur les mathématiques et la physique, et pas seulement sur la logique.

5. Un tel recours est revendiqué par Quine, qui écrit, dans "Le domaine et le langage de la science ", p. 227: "Quoique la science recherche des traits de la réalité indépendants de la langue, elle ne peut ni remporter de succès sans le langage, ni aspirer à la neutralité linguistique. Le savant peut, par le choix de sa langue, augmenter un peu l'objectivité et diminuer l'ingérence du langage. Quant à nous, préoccupés que nous sommes de dévoiler l'essence du discours scientifique, nous pouvons remodeler avec profit le langage de la science, au-delà de ce qu'on pourrait raisonnablement demander d'un savant en exercice. " Ce remodelage n'est autre que la transposition du discours scientifique en logique du premier ordre, que Quine décrit comme une "notation canonique : «Dans notre notation canonique de la quantification, nous trouvons que l'ordre et la légalité sont restaurés. Dans la mesure où nous nous en tenons à cette notation, les objets que nous sommes censés admettre sont précisément les objets que nous reconnaissons comme faisant partie de l'univers dans lequel les variables 
Résumons le mouvement général de la présente étude:

1) Poincaré affirme que les mathématiques sont la seule langue que le physicien puisse parler; c'est ainsi en termes de langage approprié ou non que Poincaré traite la question de la nature mathématique de la physique.

2) Par ailleurs, les écrits philosophiques de Poincaré suggèrent la thèse selon laquelle le langage ordinaire véhicule une "théorie» implicite qui est fondamentalement ontologique: c'est un langage de choses (d'entités) soumis au régime de l'identité.

3) En raison de la nature du donné brut de l'expérience (le donné sensible), la mesure des grandeurs physiques ne se prête pas au régime de l'identité, c'est-à-dire au régime ontologique ("métaphysique ", écrit Poincaré): la physique étudie non pas des entités, mais des grandeurs; le langage ordinaire est par conséquent inadéquat aux données sensibles (qui constituent la base de toute expérience physique).

4) Les analyses philosophiques déployées par Poincaré, principalement au sujet du continu mathématique et de la géométrie, visent inversement à établir leur adéquation aux caractères du donné sensible de toute expérience physique (par adéquation, il faut entendre que la géométrie et l'analyse constituent un langage approprié au donné sensible).

5) On peut alors conclure, dans le même temps:

- à la non-pertinence des considérations métaphysiques dans le cadre de la physique et des mathématiques (du moins d'une partie significative de celles-ci);

- à la nature nécessairement mathématique de la physique.

\section{Substance et identité}

Concentrant son attention sur le noyau mathématique des théories physiques et affirmant que relèvent de la métaphysique certains questionnements

liées de la quantification sont censées prendre leurs valeurs. Tel est précisément le sens que nous entendons donner aux quantificateurs « $(x) »$ et « $\$ x »$; « tout objet $x$ est tel que ", « il existe un objet $x$ tel que ". Les quantificateurs sont des manières d' «encapsuler " ces constructions choisies du langage ordinaire qui servent sans équivoque à renvoyer à des objets. Paraphraser une phrase dans la notation canonique de la quantification, c'est, d'abord et pardessus tout, expliciter son contenu ontique, la quantification étant un procédé pour parler des objets en général." (Quine, Le Mot et la chose, p. 335). Comme ce dernier texte l'illustre bien, la clarification recherchée est principalement d'ordre ontologique. Nous allons voir que c'est précisément la pertinence d'une telle recherche qui est critiquée par Poincaré. Ce n'est pas la solidarité entre logique et ontologie, affirmée par Quine, qui sépare celui-ci de Poincaré, bien au contraire : c'est précisément en raison de cette solidarité que, lorsque Poincaré dénonce comme dénués de sens les questionnements ontologiques relatifs aux théories scientifiques, il interdit aussi par là même que l'analyse logique puisse constituer un instrument de compréhension philosophique de ces théories. 
et certaines hypothèses ${ }^{6}$ de nature ontologique qui n'ont pas de liens effectifs avec ce noyau, Poincaré est naturellement conduit à dénoncer l'usage de la notion de substance en physique. L'objet de cette première partie est de montrer qu'à travers cette critique, Poincaré cherche à mettre en évidence une distinction fondamentale entre le langage mathématique et le langage ordinaire, en dégageant ce que nous appellerons la constitution ontologique de celui-ci.

\subsection{Le statut du langage dans la philosophie de Poincaré}

C'est en termes de langue appropriée que Poincaré affirme à plusieurs reprises la nature nécessairement mathématique de la physique. En témoignent les citations suivantes:

Les théories mathématiques n'ont pas pour objet de nous révéler la véritable nature des choses; ce serait là une prétention déraisonnable. Leur but unique est de coordonner les lois physiques que l'expérience nous fait connaitre, mais que sans le secours des mathématiques nous ne pourrions même énoncer ${ }^{7}$.

Toutes les lois sont tirées de l'expérience, mais pour les énoncer, il faut une langue spéciale; le langage ordinaire est trop pauvre, il est d'ailleurs trop vague, pour exprimer des rapports si délicats, si riches et si précis.

Voilà donc une première raison pour laquelle le physicien ne peut se passer des mathématiques; elles lui fournissent la seule langue qu'il puisse parler ${ }^{8}$.

6. Il s'agit de ce que Poincaré appelle les «hypothèses indifférentes", qui n'ont de vertu qu'heuristique et qui consistent en fin de compte à «habiller» de langage ordinaire (ce qui, nous allons le voir, explique leur caractère ontologique) le noyau mathématique des théories physiques: "Les hypothèses de ce genre n'ont donc qu'un sens métaphorique. Le savant ne doit pas plus se les interdire, que le poète ne s'interdit les métaphores; mais il doit savoir ce qu'elles valent. Elles peuvent être utiles pour donner une satisfaction à l'esprit, et elles ne seront pas nuisibles pourvu qu'elles ne soient que des hypothèses indifférentes.

Ces considérations nous expliquent pourquoi certaines théories, que l'on croyait abandonnées et définitivement condamnées par l'expérience, renaissent tout à coup de leurs cendres et recommencent une vie nouvelle. C'est qu'elles exprimaient des rapports vrais; et qu'elles n'avaient pas cessé de le faire quand, pour une raison ou pour une autre, nous avions cru devoir énoncer les mêmes rapports dans un autre langage." (Poincaré, La Science et l'hypothèse, p. 176.) Ainsi en est-il par exemple de la théorie de Fresnel dans laquelle tel vecteur est représenté par un mouvement, alors que le même vecteur devient un courant dans la théorie de Maxwell.

7. Poincaré, La Science et l'hypothèse, p. 215. Remarquons qu'au caractère mathématique du langage de la physique, sont ici associés à la fois la dimension expérimentale de la physique et le questionnement métaphysique (qui a pour objet «la véritable nature des choses»). Nous pouvons résumer la présente étude en disant qu'elle a pour objet de comprendre la signification de cette triple association.

8. Poincaré, La Valeur de la science, p. 105. 
En écrivant: "la seule langue », Poincaré semble ici suggérer qu'il existe une différence fondamentale entre le langage ordinaire et le langage mathématique (ou plus précisément les mathématiques comme langage). Avant d'essayer de mettre en évidence cette différence, il convient de préciser le statut du langage au sein de la philosophie de Poincaré9. Les indications qui suivent ne prétendent pas fournir un exposé complet et raisonné de la conception poincaréienne du langage; il s'agit seulement de donner quelques précisions qui seront utiles pour la suite de l'étude.

Par langue ou langage, Poincaré entend de façon très générale un système de pensée qui ne se réduit:

a) ni à un système de signes;

b) ni à un système de représentation.

a) Dire que la mathématique est un langage - celui de la physique n'implique pas, pour Poincaré, que les mathématiques sont un système de signes. Par exemple, les diverses intuitions qui font partie intégrante de la mathématique sont par là même constitutives du langage que sont les mathématiques, sans pour autant pouvoir être comprises comme des signes.

b) Poincaré partage avec le pragmatisme et l'idéalisme ${ }^{10}$ le refus de réduire la pensée (et par conséquent le langage) à un système de représentation:

Tout ce qui n'est pas pensée est le pur néant; puisque nous ne pouvons penser que la pensée et que tous les mots dont nous disposons pour parler des choses ne peuvent exprimer que des pensées; dire qu'il y a autre chose que la pensée, c'est donc une affirmation qui ne peut avoir de sens ${ }^{11}$.

Dans l'extrait tiré de l'article «Les mathématiques et la logique » cité dans la note précédente, Poincaré caractérise le pragmatisme et l'idéalisme comme des conceptions opposées au réalisme, lequel se définit comme l'affirmation de l'existence de choses indépendantes de la pensée. S'op-

9. Nous serons conduit dans ce paragraphe à anticiper quelque peu sur des développements qui prendront leur place plus loin; il est difficile de faire autrement dans l'exposé d'une pensée comme celle de Poincaré, qui se décline selon de nombreux thèmes tous solidaires et dont le style n'est pas celui de l'argumentation linéaire.

10. Pragmatisme et idéalisme, pour lesquels Poincaré prend explicitement parti en divers textes, sont associés et identifiés dans «Les mathématiques et la logique »: "Ceux que j'ai appelés les pragmatistes sont idéalistes, les cantoriens sont des réalistes. [...] [les pragmatistes] considèrent qu'un objet n'existe que quand il est pensé, et qu'on ne saurait concevoir un objet pensé indépendamment d'un sujet pensant. C'est bien là de l'idéalisme. [...] Les réalistes se placent d'ordinaire au point de vue physique [«réalisme physique»]; ce sont les objets matériels, ou les âmes individuelles, ou ce qu'ils appellent les substances, dont ils affirment l'existence indépendante. [...] Cela, c'est le point de vue du sens commun, et ce n'est que par la réflexion qu'on peut être amené à l'abandonner." (Poincaré, Dernières Pensées, p. 157-159.)

11. Poincaré, La Valeur de la science, p. 187. 
posant à une telle conception, Poincaré ne peut pas considérer que le langage et la pensée sont des systèmes de représentation, car une telle vue suppose que soit donné indépendamment d'eux ce qu'ils seraient censés représenter. Si l'idéalisme de Poincaré n'exclut pas la reconnaissance d'un donné $^{12}$, il implique ainsi que tout langage contient en lui-même quelque chose comme une théorie plus ou moins implicite:

Chacun porte en soi sa conception du monde dont il ne peut se défaire si aisément. Il faut bien, par exemple, que nous nous servions du langage, et notre langage n'est pétri que d'idées préconçues et ne peut l'être d'autre chose ${ }^{13}$.

Ainsi, tout énoncé est dans une certaine mesure constitutif de ce dont il parle, et le mode de cette constitution est déterminé par le langage dans lequel l'énoncé est formulé.

Ce qui est suggéré par Poincaré dans les textes cités plus haut peut donc être formulé comme suit: il y a une différence fondamentale entre les théories implicites véhiculées par le langage ordinaire et par le langage mathématique qui expliquerait la nature nécessairement mathématique de la physique.

\subsection{Langage ordinaire et ontologie}

Dans un texte cité plus haut, Poincaré écrit: «Les théories mathématiques n'ont pas pour objet de nous révéler la véritable nature des choses ${ }^{14} »$. Ce qui est ici visé correspond à ce que Poincaré appelle "métaphysique». Poincaré renvoie régulièrement à la métaphysique ce type de questions:

Entre toutes ces explications [mécaniques] possibles [des phénomènes électromagnétiques], comment faire un choix pour lequel le secours de l'expérience nous fait défaut? Un jour viendra peut-être où les physiciens se désintéresseront de ces questions, inaccessibles aux méthodes positives, et les abandonneront aux métaphysiciens. Ce jour n'est pas venu; l'homme ne se résigne pas si aisément à ignorer éternellement le fond des choses ${ }^{15}$.

Peu nous importe que l'éther existe réellement, c'est l'affaire des métaphysiciens ${ }^{16}$.

12. Il convient en effet de nuancer l'affirmation donnée dans les dernières lignes de $L a$ Valeur de la science citées ici. Selon Poincaré, il y a (bien qu'il soit impropre de le dire ainsi) un autre de la pensée et du langage: il s'agit du donné sensible lui-même. Nous verrons que c'est précisément en référence au donné sensible que les mathématiques (plus précisément: l'analyse et la géométrie) seront décrites comme le langage adéquat de la physique, ce qui ne signifie pas qu'elles permettent de représenter les sensations, mais qu'elles fournissent des cadres adéquats par lesquels est rendue possible la mesure, laquelle n'est pas une représentation, ne serait-ce que des rapports entre les sensations, mais une opération qui s'effectue au sein même des sensations, ou, pour le dire mieux: une opération entre des ensembles de sensations, dont l'ordonnancement est la vocation fondamentale de la géométrie.

13. Poincaré, La Science et l'hypothèse, p. 159.

14. Ibid., p. 215.

15. Ibid., p. 225.

16. Ibid., p. 215. 
Il peut se faire [en cas de contradiction entre deux théories physiques] qu'elles expriment l'une et l'autre des rapports vrais et qu'il n'y ait de contradiction que dans les images dont nous avons habillé la réalité.

À ceux qui trouvent que nous restreignons trop le domaine accessible au savant, je répondrai: Ces questions, que nous nous interdisons et que vous regrettez, ne sont pas seulement insolubles, elles sont illusoires et dépourvues de sens ${ }^{17}$.

Il est important de noter que les questions métaphysiques ne sont pas dépourvues de sens en elles-mêmes, mais en tant qu'on attend des théories scientifiques qu'elles y répondent. Nous allons voir que ce que Poincaré affirme ici, c'est que les théories scientifiques ne peuvent répondre à ces questions, non pas par limitation ou impuissance, mais parce que ces questions sont formulées dans un langage qui n'est pas celui de ces théories (c'est pour cela qu'elles sont, dans ce contexte, dépourvues de sens). Comme le montrent les textes cités ci-dessus, ces questions sont de nature ontologique, au sens où ce sont des questions d'existence ou d'essence.

Comme cela arrive fréquemment sous la plume de Poincaré, un même terme peut prendre plusieurs significations selon le contexte dans lequel il est utilisé. C'est le cas du terme "métaphysique ", qui désigne, d'une part, de façon très générale, la tradition philosophique, d'autre part le questionnement d'ordre ontologique, entendu comme questionnement relatif à l'existence et à l'essence d'entités censées constituer la réalité ultime, «le fond des choses". Ces deux acceptions du terme ne doivent pas être confondues, dans la mesure où c'est spécifiquement selon la seconde que l'on trouve chez Poincaré une critique de la métaphysique, ou plus précisément une critique des questionnements métaphysiques adressés aux théories physiques. Ainsi, lorsque Poincaré parle de la notion métaphysique du continu (d'ailleurs associé au continu des physiciens), qu'il oppose à la définition mathématique du continu telle qu'elle est donnée par les analystes ${ }^{18}$, il n'entend nullement dénoncer la première comme dénuée de sens, ou même seulement comme non pertinente en philosophie des mathématiques. Nous y reviendrons.

Ce n'est donc pas tant la métaphysique en tant que telle qui est critiquée par Poincaré que la tendance à développer une lecture ontologique des théories scientifiques. Or, la métaphysique entendue comme souci ontologique semble constituer précisément un aspect de la théorie implicite véhiculée par le langage ordinaire. Ceci est manifeste dans la suite d'un texte cité plus haut:

[...] le physicien ne peut se passer des mathématiques; elles lui fournissent la seule langue qu'il puisse parler.

17. Ibid., p. 175 .

18. Ibid., p. 47, 48. 
Et ce n'est pas une chose indifférente qu'une langue bien faite; pour ne pas sortir de la physique, l'homme inconnu qui a inventé le mot chaleur a voué bien des générations à l'erreur. On a traité la chaleur comme une substance, simplement parce qu'elle était désignée par un substantif, et on l'a crue indestructible.

En revanche, celui qui a inventé le mot électricité a eu le bonheur immérité de doter implicitement la physique d'une loi nouvelle, celle de la conservation de l'électricité, qui, par un pur hasard, s'est trouvée exacte, du moins jusqu'à présent ${ }^{19}$.

On peut faire ici deux remarques:

1) Il y est question de la substance, notion centrale de l'ontologie ${ }^{20}$; et Poincaré affirme ici conjointement:

- l'usage nécessaire du langage mathématique en physique;

- le danger et l'inutilité de l'usage de cette notion en physique ${ }^{21}$.

2) Poincaré s'appuie sur une considération linguistique: c'est parce que nous utilisons des substantifs que nous sommes conduits à considérer ce dont nous parlons comme des substances (l'accent étant mis ici sur le caractère de permanence, de subsistance, attaché à la notion de substance).

Ce qui apparaît ici n'est donc en fait rien d'autre qu'un aspect de la théorie implicite véhiculée par le langage ordinaire: c'est un langage ontologique, au sens où il véhicule, dans sa grammaire même, la notion de substance (qui appartient au réseau conceptuel de l'existence et de l'essence). Dès lors, l'hypothèse suivante peut être tirée des deux remarques formulées cidessus: si la mathématique est la seule langue que le physicien puisse parler, c'est parce que, contrairement au langage ordinaire, elle n'est pas ontologique et fonctionne donc selon un mode différent de celui de la métaphysique de la substance. Ainsi, si les questions métaphysiques sont dénuées de sens dès lors qu'elles sont posées aux théories physiques, c'est parce qu'elles ressortissent à la grammaire ontologique du langage ordinaire, laquelle constitue précisément ce qui distingue celui-ci du langage mathématique.

Avant de préciser davantage le sens de cette suggestion, remarquons qu'on peut la reconnaître dans ce que Poincaré annonce comme la thèse centrale de La Science et l'hypothèse:

ce que [la science] peut atteindre, ce ne sont pas les choses elles-mêmes, comme le pensent les dogmatistes naifs, ce sont seulement les rapports entre les choses; en dehors de ces rapports, il n'y a pas de réalité connaissable ${ }^{22}$.

19. Poincaré, La Valeur de la science, p. 105.

20. Une autre notion renvoyée régulièrement par Poincaré à la métaphysique est celle de cause. Nous n'en parlerons pas ici.

21. Bachelard y verra un «obstacle épistémologique »; Poincaré y souscrirait certainement, mais ce n'est pas fondamentalement selon cette perspective qu'il s'intéresse à la notion de substance.

22. Poincaré, La Science et l'hypothèse, p. 25. 
Poincaré affirmant par ailleurs que "[les] mathématiciens n'étudient pas des objets, mais des relations entre les objets ${ }^{23}$ » («chose» et «objet » ayant ici même signification), il en résulte, en vertu des développements précédents, qu'il s'agit ici, non pas d'énoncer la thèse d'un réalisme structural qui consisterait en fin de compte à substantifier les relations, mais au contraire de souligner que la réalité que la physique donne à connaître n'est précisément pas de même nature que celle qui est affirmée par le réaliste, à savoir une réalité conçue en termes d'existence de choses. La question n'est pas pour Poincaré de savoir si ce sont des choses ou des rapports entre les choses qui possèdent un mode d'existence indépendant, mais de souligner que l'erreur, lorsqu'on réfléchit sur la physique, est justement de comprendre la réalité selon le mode de l'existence indépendante, c'est-à-dire selon la grammaire de la substance. Autrement dit, Poincaré ne cherche pas à dire que ce dont le réaliste affirme l'existence n'existe $\operatorname{pas}^{24}$ et à lui substituer une autre sorte d'entités (les relations), mais à faire valoir que le réalisme (qu'il soit des choses ou des relations) est dépendant d'un mode de discours (que nous avons appelé "ontologique») qui est celui du langage ordinaire et qui ne peut être celui de la physique. Lorsque Poincaré affirme que la seule réalité connaissable (par la science physique) est constituée de rapports entre les choses et non de choses, il s'agit précisément pour lui de remarquer que les rapports en question (qui sont en fait de nature mathématique) ne doivent pas être compris selon la logique de la chose (de la substance): ce ne sont pas des entités; et que la réalité que donne à connaître la physique ne doit donc pas être conçue comme relevant de l'ontologie de la substance, laquelle est impliquée dans la position réaliste telle que Poincaré la définit. Si la formulation de cette thèse prête à une interprétation en termes de réalisme structural, c'est peutêtre parce que, elle-même formulée en langage ordinaire, elle cherche à dire un trait du langage mathématique qui, précisément, le distingue du langage ordinaire $^{25}$.

23. Ibid., p. 49.

24. Ce qui, comme le souligne Quine dans les premières lignes de "On what there is", constitue une critique autodestructrice du réalisme.

25. La critique de l'interprétation de la philosophie de Poincaré comme réalisme structural, défendue notamment par Giedymin et E. Zahar, ne saurait se réduire à cette remarque et appellerait une longue discussion. Nous voulons seulement ici suggérer que ce ne saurait être en suivant strictement la caractérisation poincaréienne du réalisme qu'une telle caractérisation de cette philosophie pourrait être poursuivie. Il n'est pas inutile ici de donner de nouveau le texte extrait de "Les mathématiques et la logique »: "Ceux que j'ai appelés les pragmatistes sont idéalistes, les cantoriens sont des réalistes. [...] [les pragmatistes] considèrent qu'un objet n'existe que quand il est pensé, et qu'on ne saurait concevoir un objet pensé indépendamment d'un sujet pensant. C'est bien là de l'idéalisme. [...] Les réalistes se placent d'ordinaire au point de vue physique ["réalisme physique»]; ce sont les objets matériels, ou les âmes individuelles, ou ce qu'ils appellent les substances, dont ils affirment l'existence indépendante. [...] Cela, c'est le point de vue du sens commun, et ce n'est que par 
Ainsi, l'opposition poincaréienne au réalisme ne saurait s'inscrire dans un débat du type: réalisme versus nominalisme, qui porte sur la nature des entités, c'est-à-dire des choses dont il est légitime ou non d'affirmer l'existence. C'est en effet la question même de savoir ce qui existe qui apparaît, aux yeux de Poincaré, comme non pertinente en philosophie des sciences.

L'idée développée par Poincaré semble donc être la suivante: le langage mathématique n'est pas un langage de choses, étant entendu que l'on conçoit par là des substances, des entités, ce qui implique des caractères qui ne sont pas indifférents, notamment, on l'a vu, la notion de permanence, mais aussi celle d'identité numérique, à laquelle la suite de ce travail est consacrée, et à partir de laquelle nous allons tâcher de confirmer les hypothèses interprétatives exposées ci-dessus.

\subsection{Identité et existence}

Dénonçant toujours l'usage de la notion de substance en physique, Poincaré fait en effet référence à une seconde détermination de cette notion, dont nous allons voir qu'elle a trait à l'identité numérique ${ }^{26}$ :

Les anciens fluides, calorique, électricité, etc., ont été abandonnés quand on s'est aperçu que la chaleur n'est pas indestructible. Mais ils l'ont été aussi pour une autre raison. En les matérialisant, on accentuait pour ainsi dire leur individualité, on creusait entre eux une sorte d'abîme. Il a bien fallu le combler quand on a eu un sentiment plus vif de l'unité de la nature, et qu'on a aperçu les relations intimes qui en relient toutes les parties ${ }^{27}$.

Le second caractère de la notion de substance retenu par Poincaré apparaît donc être la séparation, la différence ou la diversité. Notons qu'elle est solidaire de l'idée d'existence indépendante possible évoquée plus haut. Lorsque Poincaré écrit que les substances sont séparées parce qu'individuées, il ne fait que rappeler la détermination la plus fondamentale de la notion de substance, nécessaire à tout ce dont cela a un sens d'affirmer l'existence (indépendamment du fait que ce soit vrai ou faux), à savoir qu'une substance est numériquement une, comme l'exprime une formule

la réflexion qu'on peut être amené à l'abandonner. (Poincaré, Dernières Pensées, p. 157159.) Suggérons que si le point de vue du sens commun est le réalisme, c'est essentiellement parce que le langage ordinaire est ordonné à la logique de la substance, laquelle implique qu'une chose ne peut être considérée comme réelle que si elle admet un mode d'existence indépendant (notamment du sujet pensant). À cet égard, la définition cartésienne de la substance est particulièrement éloquente: «Lorsque nous concevons la substance, nous concevons seulement une chose qui existe de telle façon qu'elle n'a besoin que de soi-même pour exister. "(Descartes, Principes de la philosophie, I, 51.)

26. Par "identité», nous entendrons toujours dans ce qui suit l'identité numérique entendue comme le caractère essentiel qu'a une chose d'être numériquement une, c'est-à-dire d'être la même qu'elle-même.

27. Poincaré, La Science et l'hypothèse, p. 180. 
célèbre de Leibniz: "Pour trancher court, je tiens pour un axiome cette proposition identique qui ne diffère que par l'accent: que ce qui n'est pas véritablement un être n'est pas non plus véritablement un $\hat{e} t r e^{28}$ ».

Or, l'individualité ainsi comprise, solidaire de la diversité et de la différence, repose sur le principe d'identité ( $A$ est $A »)$, comme le rappelle, par exemple, Husserl:

Il ne peut être question d'une quantité [au sens du nombre des objets d'une collection] que là où il y a des objets différents les uns des autres. [...] Des représentations de différence appartiennent donc d'une manière essentielle à la représentation de chaque ensemble. De plus, quand nous distinguons des autres chaque objet singulier de cet ensemble, avec la représentation de la distinction est donnée aussi en même temps d'une manière nécessaire la représentation de l'identité de chaque objet avec lui-même. Dans la représentation d'une quantité concrète, chaque objet singulier est donc pensé aussi bien comme différent de tous les autres que comme identique à luimême ${ }^{29}$.

Le second trait fondamental de la notion de substance ici relevé par Poincaré est donc celui de l'identité et de la différence, diversité ou altérité: le discours ontologique fonctionne selon le régime du même et de l'autre ${ }^{30}$. Ici encore, Quine formule de façon particulièrement claire la solidarité entre le principe d'identité et l'existence: «Point d'entité sans identitée ${ }^{31}$.»

[...] le véritable emploi de termes et la position [c'est-à-dire la position d'existence] d'objets ne sont identifiables primordialement que clavetés dans les tours de la mêmeté et de la différence. Ce qui se passe, c'est qu'au départ nous apprenons des patrons généraux de parler en termes et de parler de choses, avec l'aide des accessoires indispensables de l'identitéér ${ }^{2}$.

Lorsque nous parlons d'une chose, d'une entité, d'une substance, c'est-à-dire de quelque chose dont cela a un sens de dire qu'elle peut exister, alors est impliquée dans ce que nous disons la grammaire de l'identité et de la différence. Cela signifie que pour parler d'une entité, d'une chose, il faut que cela ait un sens de dire: "c'est la même", "c'en est une autre", etc. Encore une fois, les textes cités jusqu'ici montrent que Poincaré souscrirait sans aucun doute à ce qu'écrit ici Quine. Ce qui le sépare de ce dernier est l'idée selon laquelle le langage mathématique ne fonctionne pas selon cette logique et ne véhicule donc aucune ontologie. C'est la raison pour laquelle les questionnements ontologiques sont dépourvus de sens en ce qui concerne les sciences mathématiques, ce qui permet de comprendre que, si Quine écrit:

28. Leibniz, lettre à Arnauld du 30 avril 1687.

29. Husserl, Philosophie de l'arithmétique, p. 60.

30. Nous verrons plus bas que ce point revêt une importance fondamentale au sein des considérations de Poincaré sur la question du continu.

31. Quine, "Parler d'objets", p. 35.

32. Quine, "Parler d'objets", p. 31. 
Il y a des philosophes qui persistent à dire que le verbe "existe ", dit à propos de nombres, de classes et de choses semblables, et le verbe "existe", dit à propos d'objets matériels, sont deux usages d'un même terme ambigu "existe». Ce qui me déconcerte le plus, c'est la fermeté de leur insistance. Qu'est-ce qui pourrait tenir lieu ici de justification ou d'indice en leur faveur ${ }^{33}$ ?

Poincaré, quant à lui, affirme: "[Le] mot existence n'a pas le même sens quand il s'agit d'un être mathématique et quand il est question d'un objet matériel ${ }^{34}$.»

Tâchons à présent de montrer qu'effectivement, la conception philosophique de Poincaré implique que l'on ne peut pas, à proprement parler, considérer le langage mathématique (ou du moins une partie significative de celui-ci) comme un langage de choses (d'entités, de substances), c'est-à-dire comme un langage ontologique; c'est là le sens, selon notre lecture, de l'opposition de Poincaré à ce qu'il appelle le réalisme. Le fil conducteur de cet examen sera la notion d'identité : s'il n'y a "point d'entités sans identité ", alors, en montrant que l'interprétation du signe «= " comme signe de l'identité n'est pas pertinente, par là même sera fourni un élément en faveur de l'idée selon laquelle cela n'a pas de sens de parler d'entités mathématiques (et physiques, puisque la science physique est fondamentalement mathématique).

\section{2. Égalité et approximation}

C'est en particulier au sein de ses considérations sur la notion de continuité et sur le calcul infinitésimal que l'on peut trouver dans les écrits philosophiques de Poincaré un aspect de la thèse selon laquelle le langage mathématique ne ressortit pas, contrairement au langage ordinaire, au régime ontologique de l'identité. C'est cette idée que Brunschvicg décèle au sein des œuvres fondatrices du calcul différentiel; ainsi écrit-il, au sujet de l' «adégalité» de Fermat que "cette pensée différentielle se livre à nous dans sa nudité, elle ne se réfère à aucune forme de la logique traditionnelle; l'adégalité déborde le cadre rigide du principe d'identité ${ }^{35}$ ».

33. Quine, Le Mot et la chose, p. 193.

34. Poincaré, La Science et l'hypothèse, p. 70. Rappelons que la question de l'existence des objets matériels est renvoyée par Poincaré à la métaphysique: "Peu nous importe que l'éther existe réellement, c'est l'affaire des métaphysiciens; l'essentiel pour nous c'est que tout se passe comme s'il existait et que cette hypothèse est commode pour l'explication des phénomènes. Après tout, avons-nous d'autre raison de croire à l'existence des objets matériels? » (Poincaré, La Science et l'hypothèse, p. 215.) Il faut noter ici que l'usage effectif de la notion d'éther en physique est en réalité indépendant de la stricte assomption de son existence, assomption métaphysique qui, en tant que telle, est une hypothèse indifférente. Sauf à se contredire, Poincaré n'accorde donc pas au "tout se passe comme s'il existait» un sens ontologique! Toutefois, ce texte indique que la considération des objets matériels (dits aussi "corps extérieurs»), du point de vue de leur existence et, partant, en tant qu'entités individuées ou substances, relève, pour Poincaré, de la métaphysique.

35. Brunschvicg, Les Étapes de la philosophie mathématique, p. 179. 
C'est par ailleurs en référence au principe d'identité que l'on peut lire ce que Poincaré présente comme un problème philosophique majeur dans le cadre de la question de la nature mathématique de la physique. Ce problème porte sur la notion de continuité et est posé par Poincaré à plusieurs reprises. La formulation la plus explicite de ce problème se trouve dans l'article intitulé "Cournot et les principes du calcul infinitésimal»:

[...] le jour où l'on a défini le nombre incommensurable d'une façon satisfaisante, de façon à parfaire ce que l'on avait appelé l'arithmétisation de l'analyse mathématique, les derniers voiles ont été levés, à tel point que nous avons aujourd'hui peine à comprendre ce qui a pu autrefois paraître obscur.

Est-ce à dire que l'étude des difficultés aujourd'hui vaincues, et des efforts qu'on a faits pour lutter contre elles, soit désormais dépourvue de tout intérêt ou n'ait plus qu'un intérêt historique? Il s'en faut de beaucoup; il semble qu'en s'arithmétisant, en s'idéalisant pour ainsi dire, la mathématique s'éloignait de la nature et le philosophe peut toujours se demander si les procédés du calcul différentiel et intégral, aujourd'hui justifiés au point de vue logique, peuvent être légitimement appliqués à la nature. Le continu que nous offre la nature et qui est en quelque sorte une unité est-il semblable au continu mathématique, tel que l'ont défini les plus récents géomètres, et qui n'est plus qu'une multiplicité d'éléments, en nombre infini, mais extérieurs les uns aux autres et pour ainsi dire logiquement discrets ${ }^{36}$ ?

C'est également comme traitant de ce même problème que Poincaré présente, dans l'introduction de La Science et l'hypothèse, le chapitre consacré à la grandeur continue:

[...] trouvons-nous [la grandeur mathématique — c'est-à-dire le continu mathématique ${ }^{37}$ ] dans la nature, ou est-ce nous qui l'y introduisons ? Et, dans ce dernier cas, ne risquons-nous pas de tout fausser? Comparant les données brutes de nos sens et ce concept extrêmement complexe et subtil que les mathématiciens appellent grandeur, nous sommes bien forcés de reconnaitre une divergence; ce cadre où nous voulons tout faire rentrer, c'est donc nous qui l'avons fait; mais nous ne l'avons pas fait au hasard, nous l'avons fait pour ainsi dire sur mesure et c'est pour cela que nous pouvons y faire rentrer les faits sans dénaturer ce qu'ils ont d'essentiel ${ }^{38}$.

Notons que Poincaré ajoute ici une suggestion significative: non seulement le continu mathématique appelle une élucidation philosophique pour comprendre son application à la connaissance de la nature, mais cette application est sa vocation primordiale. La divergence mentionnée dans ce texte est décrite, au sein du chapitre, selon les mêmes termes que dans l'article consacré à Cournot:

36. Poincaré, L'Opportunisme scientifique, p. 107.

37. Le contenu du deuxième chapitre de La Science et l'bypothèse ne laisse aucun doute sur cette identification. Rappelons en outre que le titre initial de l'article dont est tiré ce chapitre était «Le continu mathématique».

38. Poincaré, La Science et l'hypothèse, p. 25. 
Le continu ainsi conçu [de manière ensembliste, comme le fait Dedekind par exemple] n'est plus qu'une collection d'individus rangés dans un certain ordre, en nombre infini, il est vrai, mais extérieurs les uns aux autres. [...] De la célèbre formule, le continu est l'unité dans la multiplicité, la multiplicité seule subsiste, l'unité a disparu. Les analystes n'en ont pas moins raison de définir leur continu comme ils le font, puisque c'est toujours sur celui-là qu'ils raisonnent depuis qu'ils se piquent de rigueur ${ }^{39}$.

La proximité entre ces formulations et celles des textes, cités plus haut, consacrés aux "anciens fluides ${ }^{40}$ " et au réalisme ${ }^{41}$ invite à comprendre que le caractère "logiquement discret» d'individus extérieurs les uns aux autres ne semble pouvoir renvoyer à autre chose qu'à l'altérité ou diversité ontologique, dont nous avons vu qu'elle est solidaire de l'identité à soi-même de toute substance individuée. La suggestion suivante peut dès lors être proposée: si l'on comprend le continu mathématique selon la logique de l'identité, alors il n'est pas possible de comprendre son rôle privilégié dans la connaissance de la nature, c'est-à-dire en physique; la contraposée de cette implication prend évidemment la forme suivante: la physique n'est possible que formulée dans un langage qui n'est pas présidé par le principe d'identité.

\subsection{Rigueur, logique et théorie des ensembles}

Il convient d'appuyer quelque peu le rapprochement fait ci-dessus entre la description que fait Poincaré du continu mathématique «arithmétisé » et le caractère constitutif de la notion de substance que nous avons décrit à l'aide du couple identité/diversité. Nous avons insisté sur le fait que le caractère d'altérité (indissociable de l'identité numérique) ou d'extériorité, pour reprendre le terme choisi par Poincaré, apparaît comme l'un des éléments fondamentaux de tout discours ontologique et avons tâché de montrer que c'est notamment cette dimension ontologique que vise Poincaré lorsqu'il refuse la pertinence du discours substantialiste dans le cadre de la physique.

Remarquons à présent que la notion d'ensemble semble précisément impliquer un principe d'identité et de diversité, c'est-à-dire un principe d'individuation de ses éléments; c'est ce qu'exprime le texte de Husserl cité plus haut. Par ailleurs, il est important de souligner que c'est en tant qu'il est conçu selon les définitions résultant de l'effort de rigueur entrepris au

39. Ibid., p. 48.

40. «Les anciens fluides, calorique, électricité, etc., ont été abandonnés quand on s'est aperçu que la chaleur n'est pas indestructible. Mais ils l'ont été aussi pour une autre raison. En les matérialisant, on accentuait pour ainsi dire leur individualité, on creusait entre eux une sorte d'abîme. " (Poincaré, La Science et l'hypothèse, p. 180.)

41. "Les réalistes se placent d'ordinaire au point de vue physique; ce sont les objets matériels, ou les âmes individuelles, ou ce qu'ils appellent les substances, dont ils affirment l'existence indépendante.» (Poincaré, Dernières Pensées, p. 159) 
XIX $X^{e}$ siècle - effort dont il reconnaît une certaine légitimité - que le continu mathématique pose pour Poincaré le problème philosophique exposé plus haut. Le continu ainsi conçu est présenté par Poincaré comme n'étant satisfaisant que du point de vue de la rigueur et de la logique. Or, en dépit de l'appellation courante d' "arithmétisation ", c'est certainement le point de vue ensembliste - manifeste dans la construction de Dedekind - inhérent aux définitions rigoureuses du continu mathématique qui intéresse ici Poincaré et qui le conduit à écrire que ce continu ainsi défini est «logiquement discret». En effet, s'il ne confond certes pas l'effort de rigueur en analyse, le logicisme de Russell et de Couturat, la théorie des ensembles de Cantor et le formalisme hilbertien, Poincaré ne les place pas moins, aux premières pages du troisième chapitre de Science et méthode, sous le signe commun d'un effort "en vue de dégager et d'isoler les éléments logiques du raisonnement mathématique ${ }^{42}$ » et retient l'implication commune du point de vue ensembliste au sein de ces entreprises. Ainsi écrit-il que «les logisticiens ne parlent que de classes et de classes de classes $^{43}$ »; et, au sujet des Fondements de la géométrie de Hilbert:

Les objets que [M. Hilbert] appelle points, droite ou plan deviennent ainsi des êtres purement logiques qu'il est impossible de se représenter. On ne saurait imaginer, sous une forme sensible, ces points qui ne sont que des systèmes de trois séries. Peu lui importe; il lui suffit que ce soient des individus et qu'il ait des règles sûres pour distinguer ces individus les uns des autres, pour établir conventionnellement entre eux des relations d'égalité ou d'inégalité et pour les transformer ${ }^{44}$.

Ici encore, la diversité des individus - nécessaire au point de vue ontologique de l'identité - est mise en avant.

Ajoutons que la position de l'infini actuel est par ailleurs présentée par Poincaré comme solidaire du point de vue ensembliste et est partie prenante dans l'affirmation du réalisme des cantoriens; nous reviendrons sur ce point ultérieurement.

\subsection{Le continu physique et la mesure}

Les descriptions générales que Poincaré donne des théories physiques sont sans ambiguïté sur le fait qu'il considère que leur noyau essentiel est constitué par les équations différentielles - lois et principes - qu'elles énoncent:

42. Poincaré, Science et méthode, p. 127.

43. Ibid., p. 162.

44. Poincaré, L'Opportunisme scientifique, p. 45. Faute d'avoir entrepris une analyse suffisante de la fin de ce texte, nous ne sommes pas parvenu, à ce jour, à déterminer si la référence faite ici à "des relations d'égalité ou d'inégalité" vient à l'appui de l'interprétation ici défendue, ou si au contraire elle s'y oppose. 
Dans la conception scientifique l'état du monde, ou d'une partie du monde regardée comme isolée, sera entièrement déterminé par les valeurs attribuées à un certain nombre de variables $x_{1}, x_{2}, \ldots, x_{n}$. La connaissance de ces valeurs nous donnera non seulement l'état du monde à l'instant envisagé, à l'instant $t$, mais encore à l'instant immédiatement postérieur $t+d t$, car ces deux états sont immédiatement reliés l'un à l'autre par une relation qui est précisément ce que l'on appelle loi, et cette relation est une équation différentielle:

$d x_{i} / d t=\varphi j\left(x_{1}, x_{2}, \ldots, x_{n}\right)^{45}$

Dans tout phénomène physique, il y a un certain nombre de paramètres que l'expérience atteint directement et qu'elle permet de mesurer. Je les appellerai les paramètres $q$.

L'observation nous fait ensuite connaître les lois de variations de ces paramètres et ces lois peuvent généralement se mettre sous la forme d'équations différentielles qui lient entre eux les paramètres $q$ et le temps ${ }^{46}$.

Comme l'indique ce texte, la dimension expérimentale de la science physique, sur laquelle Poincaré insiste constamment, implique que les paramètres intervenant dans les lois sont des grandeurs mesurées expérimentalement ou susceptibles de l'être. Or, le donné brut sensible de toute expérience, sur lequel porte la mesure, n'est autre que ce que Poincaré appelle le continu physique ${ }^{47}$. L'articulation entre la question de la nature mathématique de la physique et le problème du lien entre continu physique et continu mathématique en découle.

Cette attention de Poincaré à la fonction centrale des grandeurs au sein des théories physiques participe aussi de sa défiance envers les questionnements de nature ontologique, et ce, sous au moins deux aspects. Premièrement, considérer ces grandeurs comme des propriétés ou des attributs d'entités, ou même comme les coordonnées de leur position dans un certain référentiel relève, selon Poincaré, d'un point de vue métaphysique. Le chapitre XII de La Science et l'hypothèse illustre parfaitement cette vue ${ }^{48}$. Nous ne développerons pas ce premier aspect, notre objet étant ici de mettre en évidence le second, à savoir que cela n'aurait pas de sens de considérer ces grandeurs elles-mêmes comme des entités.

Notons en outre que l'insistance de Poincaré sur le fait que les équations de la physique mettent en relation des grandeurs et la résolution qu'il prend de ne considérer que la nature mathématique de celles-ci a pour corollaire ce que l'on pourrait décrire comme une réduction de la dimension conceptuelle ${ }^{49}$ de la physique. Ainsi, par exemple, la lecture poin-

45. Ibid., p. 108.

46. Poincaré, La Science et l'hypothèse, p. 219.

47. Cette identification n'est pas exacte: donné sensible et continu physique sont à la vérité distingués par Poincaré. Nous reviendrons plus bas sur cette distinction qui n'est pas ici déterminante; jusque-là, nous entendrons abusivement par "continu physique " le donné sensible, ce que Poincaré fait aussi à l'occasion.

48. Voir plus bas, note 57 .

49. Si l'on entend par "concept physique» autre chose qu'un concept ou objet mathématique. Si nous utilisons ici l'expression "objet mathématique ", comme le fait aussi 
caréienne du principe $" F=m g$ » ne consiste pas à dire qu'il exprime une identité conceptuelle entre le concept de force et celui du produit de la masse et de l'accélération, mais seulement à conclure par exemple que «les masses sont des coefficients qu'il est commode d'introduire dans les cal$\mathrm{culs}^{50}{ }^{5}$. Si le contexte est ici celui du caractère conventionnel des principes, il n'en va pas autrement, en ce qui concerne la question des concepts physiques, pour les lois expérimentales: elles expriment fondamentalement des relations entre des grandeurs, non des identités conceptuelles ${ }^{51}$.

Le lien entre la mesure et le continu physique est l'un des thèmes constants et centraux des considérations philosophiques de Poincaré sur la géométrie et le continu mathématique ${ }^{52}$. Ainsi, le choix conventionnel du groupe des déplacements définissant une géométrie, qui s'opère à partir de l'expérience du continu physique, peut être décrit comme le choix d'un système de mesure. Donnons-en un aperçu schématique: mesurer une longueur consiste à comparer un corps donné avec un corps solide (une règle) utilisé comme étalon; cette comparaison consiste en l'application de la règle sur le corps à mesurer; cette opération suppose que soit déterminé un groupe de déplacements laissant la règle invariante (solide); mais, comme le remarque Poincaré, le continu physique ne nous donne que des ensembles mouvants de sensations, dont les changements ne permettent, en eux-mêmes, ni de distinguer entre ce que nous appelons "déplacements » et «changements d'état ", ni de discerner des «corps solides ", car tout cela n'a de sens qu'une fois que l'expérience est conçue spatialement, c'est-àdire une fois qu'est instituée la géométrie mise en œuvre; à partir du donné brut sensible, c'est l'expérience de la «compensation» de certains changements subis par des ensembles de sensations (visuelles et tactiles) par une activité musculaire volontaire (qui est aussi donnée sous la forme d'ensembles de sensations) qui suggère le choix de ce que nous appelons les «déplacements"; ce choix est en outre soumis à la condition que l'ensemble des déplacements forme un groupe; les ensembles de sensations compensés par les déplacements sont donc à peu près invariants par les transformations du groupe choisi (celui-ci a précisément été choisi pour cela) et nous appelons corps solides les ensembles de sensations invariants par le groupe; un corps solide peut alors être choisi comme règle de mesure. Chaque géométrie, c'est-à-dire chaque groupe de transformation, induit donc une assignation différente des changements du continu physique en déplacements et en changements d'état et, par suite, une sélection différente des

Poincaré, il va sans dire que nous ne l'identifions pas à «entité mathématique», avec les déterminations ontologiques, relevées plus haut, que cette expression implique.

50. Poincaré, La Science et l'hypothèse, p. 123.

51. Karine Chemla a attiré notre attention sur la question de l'identité conceptuelle. Qu'elle en soit ici remerciée. La brève remarque que nous avons consacrée à cette question ne saurait bien sûr l'épuiser. 
ensembles de sensations qui seront considérés comme correspondant à des corps solides. Le choix de la géométrie (euclidienne, elliptique ou hyperbolique) est guidé - mais non imposé - par les expériences de compensation: nous pourrions très bien choisir le langage de la géométrie hyperbolique, mais il serait moins commode pour notre expérience, car nous n'aurions alors affaire qu'exceptionnellement à des corps solides au sein de cette expérience. Toute mesure expérimentale est ainsi originellement liée aux expériences de compensations d'ensembles de sensations donnés au sein du continu physique.

L'expérience de la compensation, qui occasionne ${ }^{53}$ la mise en œuvre de la catégorie a priori de groupe de transformation, est centrale dans l'examen que nous poursuivons ici, dans la mesure où elle semble faire appel à des opérations d'identification, voire d'individuation, et ce, à deux niveaux, souvent mêlés au sein des analyses de Poincaré: 1) celui du discours commun portant sur les objets de l'expérience, c'est-à-dire les "corps extérieurs", formulé en langage ordinaire; 2) celui, plus fondamental parce que rendant possible le premier et parce seul impliqué dans la physique, de la géométrie.

Au premier niveau, l'expérience de la compensation des ensembles de sensations est solidaire de l'individuation et de l'identification des corps solides perçus lors de l'expérience commune: c'est ainsi que nous pouvons dire, à l'occasion d'un certain changement survenu au sein du continu physique, que c'est le même corps qui s'est déplacé, corps dont nous disons, en vertu du point de vue réaliste du sens commun, qu'il est la cause des divers ensembles de sensations (des divers aspects) que nous lui associons. De ce point de vue, la possibilité de la compensation résulte du caractère solide du corps en question:

Pour que la compensation se produise, il faut évidemment que les diverses parties de l'objet extérieur d'une part, les divers organes de nos sens d'autre part, se retrouvent après le double changement, dans la même position relative. Et pour cela il faut que les diverses parties de l'objet extérieur aient également conservé, les unes par rapport aux autres, la même position relative et qu'il en soit de même des diverses parties de notre corps les unes par rapport aux autres ${ }^{54}$.

Il est crucial de remarquer que ce discours du sens commun - qui relève fondamentalement, pour Poincaré, du registre métaphysique en ce

52. Ce qui confirme, étant donné que ces considérations constituent une forte proportion des écrits philosophiques de Poincaré consacrés aux mathématiques, l'importance pour celui-ci de la question de la nature mathématique de la physique.

53. Nous avons affaire ici à un exemple du thème poincaréien, central au sein des considérations relatives aux conventions, que Gerhard Heinzmann caractérise comme l' «occasionnalisme» de Poincaré.

54. Poincaré, La Science et l'hypothèse, p. 84. 
qu'il implique la position d'existence des corps extérieurs ${ }^{55}$ - est rendu possible par la géométrie; Poincaré introduit en effet ce texte par: «Un esprit qui saurait déjà la géométrie raisonnerait comme suit: $[\ldots]^{56}$.» De fait, la démarche de Poincaré consiste, de façon exactement inverse, à reconstruire la notion géométrique d'espace et celles qui lui sont associées (déplacements, corps solides), à partir $d u$ seul continu physique. Ce qui est effectivement donné, c'est les ensembles de sensations; les corps ne sont qu'une construction élaborée à partir d'eux. Comme nous l'avons vu, l'institution d'une géométrie est alors celle d'un langage permettant la classification des ensembles de sensations en termes de transformations ("déplacements") et d'invariants relatifs à ces transformations. Ce langage rend possible le discours en termes d'entités individuées (les corps extérieurs) identifiées par leurs propriétés spatiales et sensibles ${ }^{57}$; mais il ne l'appelle pas nécessairement: bien au contraire, la compréhension de la physique implique précisément pour Poincaré qu'on renvoie ce discours à la métaphysique et qu'on s'en tienne strictement au langage de la géométrie $^{58}$, qui ne traite que de groupes de transformations et, le cas échéant,

55. Voir note $\mathrm{n}^{\circ} 33$. Notons que Poincaré emploie indifféremment, nous semble-t-il, les expressions "corps extérieurs", "objets extérieurs", "corps matériels", "objets matériels ".

56. Poincaré, La Science et l'hypothèse, p. 84.

57. Affirmer, comme Poincaré le fait, que la connaissance de la géométrie est une condition de possibilité du discours spatial commun porté sur l'expérience sensible et déployé en langage ordinaire peut paraître surprenante: si nous maitrisons tous ce discours, peu d'entre nous sont en revanche spécialistes des groupes de Lie. La réponse de Poincaré à cette objection peut être formulée comme suit: nous savons tous en un certain sens la géométrie euclidienne puisque nous savons nous mouvoir, manipuler et déplacer des objets, prévoir les conséquences de déplacements, etc. La géométrie n'est à l'origine que la science ayant pour objet d'établir les lois de ce savoir implicite, qui est à l'œuvre dans nos actions physiques.

58. La distinction des deux langages (mathématique versus ontologique/ordinaire) souvent entremêlés au sein même du discours des scientifiques est présente dans nombre de textes de Poincaré. On peut considérer qu'elle constitue le cœur de sa discussion sur «l'explication mécanique des phénomènes physiques " (La Science et l'hypothèse, chapitre XII) dont le mouvement consiste à mettre entre parenthèses, pour ainsi dire, les coordonnées des molécules composant une substance matérielle pour établir, à partir des seuls paramètres accessibles à l'expérience, une caractérisation purement mathématique de ce qu'est une explication mécanique, ce qui, en retour, permet de mettre en évidence le caractère artificiel, indifférent et dangereusement contraignant de la caractérisation "métaphysique» d'une explication mécanique. Sans développer davantage ce point, donnons de ce texte quelques passages significatifs à cet égard:

"Que faut-il faire pour donner une explication mécanique d'un pareil phénomène?

On cherchera à l'expliquer soit par les mouvements de la matière ordinaire, soit par ceux d'un ou de plusieurs fluides hypothétiques.

Ces fluides seront considérés comme formés d'un très grand nombre de molécules isolées $m$.

[Ces deux dernières phrases fournissent une caractérisation "métaphysique »]

Quand dirons-nous alors que nous avons une explication mécanique complète du phénomène? Ce sera d'une part quand nous connaîtrons les équations différentielles auxquelles 
d'ensembles sur lesquels agissent ces groupes. Nous allons tâcher de montrer que cette distinction des discours est fondamentale dans l'analyse philosophique développée par Poincaré au sujet du continu ${ }^{59}$.

À ce second niveau — celui de l'institution du langage géométrique — s'il n'est plus directement question d'entités individuées, il n'en reste pas moins que des procédures d'identification semblent constamment à l'œuvre. De fait, les textes de Poincaré relatifs à la reconstruction de la notion d'espace à partir du donné sensible comportent de nombreuses formules du type: "la même sensation", "le même ensemble de sensations ", «le même déplacement» (entendu comme: "un changement de sensations pouvant être compensé par le même ensemble de sensations musculaires volontaires"), "des (ensembles de) sensations identiques", "identification de deux ensembles de sensations", "identité de deux

satisfont les coordonnées de ces molécules hypothétiques $m$, équations qui d'ailleurs devront être conformes aux principes de la dynamique; et d'autre part quand nous connaîtrons les relations qui définissent les coordonnées des molécules $m$ en fonction des paramètres $q$, accessibles à l'expérience.

$[\ldots]$

On obtient ce qu'on appelle les équations de Lagrange.

Dans ces équations, les variables indépendantes sont les coordonnées des molécules hypothétiques $m$; mais je suppose maintenant que l'on prenne pour variables les paramètres $q$ directement accessibles à l'expérience.

$[\ldots]$

Les équations ainsi obtenues sont une autre forme des équations de Lagrange.

Pour former ces équations, nous n'avons pas besoin de connaître les relations qui lient les paramètres q aux coordonnées des molécules hypothétiques, ni les masses de ces molécules, ni l'expression de U en fonction des coordonnées de ces molécules. [etc.] "(Poincaré, La Science et l'hypothèse, p. 220-222).

59. Distinction que ne fait pas Janet Folina dans «Logic and intuition in Poincaré's philosophy of mathematics ", ce qui la conduit à suggérer que le rôle constitutif que joue le continu mathématique au sein de l'expérience objective consiste à fournir des dispositifs d'individuation des objets en introduisant des critères d'identité: "To fully cohere with Poincaré's prior theory of the synthetic a priori, it would be necessary to justify the claim that the intuition of continuity is somehow required for systematic thinking. We might try to argue that there is no other way for finite beings to impose a criterion of sameness on both abstract and concrete objects. I, however, have at present no idea of how such an argument should be constructed." (Janet Folina , "Logic and intuition in Poincarés philosophy of mathematics", p. 432). Si nous affirmons de même que le continu possède une fonction constitutive au sein de l'expérience physique (c'est-à-dire l'expérience mesurée), nous ne pouvons en revanche suivre le second aspect de cette interprétation dans la mesure où seule l'expérience sensible commune (c'est-à-dire formulée en langage ordinaire) - qui possède certes une certaine objectivité - appelle des procédures d'individuation, lesquelles ne sont rendues possibles qu'au prix de la précision du discours: le langage ordinaire ne peut exprimer l'expérience sensible que de façon grossière parce qu'il implique l'identification d'ensembles de sensations que distinguent des instruments de mesure plus précis. Or, le continu mathématique a précisément pour vocation de constituer le langage adéquat à l'augmentation indéfinie de la précision des mesures et, pour cette raison, ne fonctionne pas selon la grammaire de l'identité et de l'individuation, comme nous allons le voir dans la suite de ce paragraphe. 
ensembles de sensations ", etc. La description de l'expérience de la compensation semble bien impliquer un discours ne pouvant se passer de l'identité. A la vérité, de telles formulations sont souvent accompagnées d'une précision tout à fait capitale, à savoir le caractère essentiellement approximatif de ces diverses "identités ". Le texte suivant en est exemplaire:

«La classification qui précède suggère deux réflexions:

$1^{\circ} \mathrm{La}$ classification n'est pas une donnée brute de l'expérience, parce que la compensation mentionnée plus haut des deux changements, l'un interne et l'autre externe, n'est jamais effectivement réalisée. C'est donc une opération active de l'esprit qui essaie d'insérer les résultats bruts de l'expérience dans une forme préexistante, dans une catégorie. Cette opération consiste à identifier deux changements parce qu'ils possèdent un caractère commun, et cela malgré qu'ils ne le possèdent pas exactement. Néanmoins, le fait même que l'esprit ait l'occasion d'accomplir cette opération est dû à l'expérience, car l'expérience seule peut lui apprendre que la compensation s'est approximativement produite $^{60}$.»

Avant d'en venir à la démonstration du fait que, par «identité », Poincaré n'entend en réalité jamais, au sein de ces contextes, l'identité numérique (toujours entendue comme la relation qu'un individu entretient avec lui-même et qui permet de formuler des énoncés du type: "c'est la même chose") mais l'égalité, et, avant d'expliquer ce qui distingue ces deux notions, tâchons de souligner l'importance accordée par Poincaré à l'approximation.

Il est facile de déduire du texte ci-dessus le caractère approximatif de toute mesure physique: celle-ci étant opérée au sein du donné sensible par comparaison d'ensembles de sensations, elle ne dépassera jamais l'approximation inhérente aux expériences de compensation qui sont à l'origine de son institution. Nous voudrions montrer ici que cette problématique de l'approximation de la mesure physique est toujours présente dans les analyses poincaréiennes du continu physique.

Si une compensation "n'est jamais effectivement" réalisée et donc toujours approximative, c'est en raison d'un caractère irréductible du donné sensible, qui justifie d'ailleurs qu'on qualifie celui-ci de continu, et qui est exprimé par ce que Poincaré appelle «la formule du continu physique", à savoir: « $\mathrm{A}=\mathrm{B} ; \mathrm{B}=\mathrm{C} ; \mathrm{A}<\mathrm{C}$ ». Pour comprendre cette formule, il faut au préalable rappeler que le donné sensible est susceptible d'une opération, celle qui consiste à pouvoir comparer entre elles certaines sensations. Poincaré est parfaitement conscient du fait que cette capacité d'ordonner des séries de sensations relève d'une certaine spontanéité de l'esprit, et c'est la raison pour laquelle il affirme que cette capacité repose sur une "catégorie de l'entendement", qu'il nomme "continu sensible » ou «continu physique». S’il s'autorise néanmoins dans de nombreux textes,

60. Poincaré, L'opportunisme scientifique, p. 10. 
comme nous l'avons annoncé, à utiliser ces appellations pour parler du donné sensible, il faut comprendre qu'il s'agit alors du donné sensible en tant qu'il est susceptible d'être ordonné selon des séries.

Les considérations de Poincaré sur la formule du continu physique comportent d'innombrables et considérables difficultés d'interprétation ${ }^{61}$. Nous n'essaierons ici ni d'en dresser la liste ni de les examiner; il s'agira seulement de souligner l'implication de la problématique de l'approximation de la mesure au sein de ces considérations. La formule du continu physique exprime le fait que des termes contigus de ces séries sont indiscernables. Pour mettre en évidence ce fait, Poincaré a recours à des considérations relatives à la mesure (de poids, par exemple) et plus précisément à la comparaison de mesures de différentes précisions. L'affirmation de l'indiscernabilité de deux sensations A et B contiguës au sein d'une série ne peut en effet avoir de sens que si l'on dispose de deux systèmes tels que dans l'un: $\mathrm{A}=\mathrm{B}$ et dans l'autre: $\mathrm{A}<\mathrm{B}$. Ces deux systèmes ne sont autres que deux systèmes de mesure dont le second est plus précis que le premier. Il faut en effet être en mesure de distinguer A et B pour affirmer que l'on a affaire à des sensations différentes selon un certain point de vue, faute de quoi cela n'aurait pas de sens de parler d'indiscernabilité. L'établissement de la formule du continu physique n'échappe pas à cette condition: si l'on s'en tenait à un seul système (celui du corps), rien ne permettrait d'affirmer que c'est une fois $\mathrm{A}$ qui est comparé avec $\mathrm{C}$ et une autre fois $\mathrm{B}$ qui est comparé avec $\mathrm{C}$, puisque, au sein de ce système, $\mathrm{A}=\mathrm{B}$. La formule ne pourrait être que quelque chose comme: $\mathrm{A}=\mathrm{A} ; \mathrm{A}<\mathrm{C}$ », qui n'est pas contradictoire. Une réponse pourrait être que c'est précisément parce que l'on a deux expériences différentes $(\mathrm{A}<\mathrm{C}$ et $\mathrm{B}=\mathrm{C})$ que l'on distingue $\mathrm{A}$ et $\mathrm{B}$, par l'intermédiaire de leur comparaison avec $\mathrm{C}$. Mais cette réponse ne tient pas, car elle implique que $\mathrm{C}$ soit distingué de $\mathrm{A}$ et de $\mathrm{B}$; or, précisément, $C$ ne peut être distingué de $B$ au sein du système, de telle sorte que $\mathrm{B}$ disparaît à nouveau et qu'on tombe sur la formule: " $\mathrm{A}<\mathrm{C} ; \mathrm{C}=$ C». La difficulté est inhérente à la contradiction interne de la formule du continu physique: la seule formulation de cette contradiction implique que puissent être distingués trois termes qui, au sein du premier système, ne le sont pas tous deux à deux. Elle implique donc que soient considérés deux systèmes de mesure. Ajoutons que la comparaison de deux systèmes est nécessaire à l'affirmation selon laquelle aucun instrument de mesure ne parviendra jamais à résoudre la contradiction, car, comme l'écrit Poincaré:

61. Comme l'illustre à n'en pas douter la suite de cet alinéa, la formulation même de considérations sur le continu physique ne peut échapper à un embarras dont nous pouvons suggérer — c'est la thèse ici défendue — qu'il n'est pas tant dû au caractère contradictoire de la formule du continu physique qu'au fait que ce dernier est précisément inaccessible à une conceptualisation déployée en langage ordinaire. 
C'est toujours avec nos sens que nous nous servons de nos instruments; c'est avec l'œil que nous observons l'image agrandie par le microscope, et cette image doit, par conséquent toujours conserver les caractères de la sensation visuelle et par conséquent ceux du continu physique ${ }^{62}$.

Les deux systèmes en question sont ici le microscope et le corps luimême, lequel n'est jamais absent de l'opération de mesure. Autrement dit, dès lors que l'on utilise un instrument de mesure autre que le corps luimême, on a affaire à deux instruments: notre corps et l'instrument en question. La problématique de la formule du continu physique est ainsi solidaire de la question de la précision des mesures, de la comparaison entre des systèmes de mesure de précisions différentes.

Le caractère "nébuleux» (continu, au sens propre) du continu physique est donc indissociable de l'approximation essentielle et irréductible de toute mesure, au sens où si l'on fait une expérience de mesure donnant: $x=y$, il existera toujours un instrument de mesure plus précis qui donnera: $x<y$ ou $x>y$. Ce dernier instrument rencontrant à son tour ce fait, il s'intercalera toujours entre $x$ et $y$ un troisième terme $z$ qui, selon un système de mesure encore plus précis, sera tel que: $x<z ; z<y$. C'est ainsi que Poincaré reconstitue la "genèse» du continu mathématique. Dans "Des fondements de la géométrie », où Poincaré ne sépare pas, comme il le fait dans La Science et l'hypothèse, les analyses sur le continu et celles sur la géométrie, l'implication de celui-là dans celle-ci est clairement appelée par le rôle fondamental joué par la géométrie dans l'opération de mesure :

"Nous avons en nous, en puissance, un certain nombre de modèles de groupes et l'expérience nous aide seulement à découvrir lequel de ces modèles s'écarte le moins de la réalité.

CONTINUITE

Nous observons d'abord que le groupe est continu. Voyons ce que cela veut dire et comment le fait peut être établi.

[...]

Nous découvrons bientôt qu'un déplacement quelconque peut toujours être divisé en deux, trois ou un nombre quelconque de parties; je veux dire que nous pouvons toujours trouver un autre déplacement qui, répété deux, trois fois, reproduira un déplacement donné. Cette divisibilité à l'infini nous conduit naturellement à la notion de la continuité mathématique; cependant les choses ne sont pas aussi simples qu'elles le paraissent à première vue.

Nous ne pouvons pas prouver cette divisibilité à l'infini directement. Quand un déplacement est très petit, il est imperceptible pour nous. Quand deux déplacements diffèrent très peu, nous ne pouvons pas les distinguer. Si un déplacement $\mathrm{D}$ est extrêmement petit, ses multiples consécutifs seront indiscernables. Il peut arriver alors que nous ne puissions pas distinguer 9D de 10D, ni 10D de 11D, mais que nous puissions néanmoins distinguer 9D

62. Poincaré, La Science et l'hypothèse, p. 52. 
de 11D. Si nous voulions transcrire ces données brutes de l'expérience en une formule, nous écririons :

$9 \mathrm{D}=10 \mathrm{D}, 10 \mathrm{D}=11 \mathrm{D}, 9 \mathrm{D}<11 \mathrm{D}$.

Ce serait là la formule du continu physique. Mais une telle formule répugne à la raison. Elle ne correspond à aucun des modèles que nous portons en nous. Nous échappons à ce dilemme par un artifice; et à ce continu physique - ou si vous préférez à ce continu sensible qui se présente sous une forme intolérable pour nos esprits —, nous substituons le continu mathématique. Séparant nos sensations de ce quelque chose que nous appelons leur cause, nous admettons que le quelque chose en question se conforme au modèle que nous portons en nous et que nos sensations s'en écartent seulement à cause de leur grossièreté.

Le même procédé revient chaque fois que nous soumettons à la mesure les données de nos sens ${ }^{63}$;"

La solidarité entre les considérations de Poincaré sur le continu physique et celles qui concernent l'approximation irréductible de toute mesure expérimentale permettent de comprendre en quoi la définition « rigoureuse » (celle de Dedekind par exemple) du continu du second ordre (c'est-à-dire des nombres rationnels et réels) ${ }^{64}$ interdit, selon Poincaré, de résoudre la question philosophique de la nature mathématique de la physique, en l'espèce: du rôle fondamental joué en physique par le continu mathématique. Ce point fait l'objet du paragraphe suivant.

\section{II.3. Identité et égalité}

La considération ensembliste des nombres rationnels et réels, nécessaire à la définition par Dedekind des réels ${ }^{65}$, si elle résout évidemment la contradiction inhérente à la formule du continu physique, ne possède plus, en raison de son caractère "logiquement discret", le caractère "continu " (au sens propre du terme), manifesté par l'indiscernabilité de termes contigus, du continu physique. Or nous avons vu dans le paragraphe précédent que l'on ne peut pas dissocier l'opération de mesure expérimentale du caractère du continu physique: tout instrument de mesure fournit un donné qui

63. Poincaré, Des fondements de la géométrie, p. 23, 24, 25.

64. Il est remarquable de constater que Poincaré se distingue de la majorité des philosophes des sciences qui lui sont contemporains et postérieurs, en ce qu'il consacre de plus amples développements au continu du premier ordre (les nombres rationnels) qu'au continu du second ordre: l'ensemble de la discussion sur la formule du continu physique ne porte effectivement que sur le premier. Ainsi écrit-il: «Dans la manière de voir de M. Dedekind [...] à chaque mode de répartition [des nombres commensurables] correspond ainsi un nombre commensurable ou non, qui lui sert de symbole. Mais se contenter de cela, ce serait trop oublier l'origine de ces symboles; il reste à expliquer comment on a été conduit à leur attribuer une sorte d'existence concrète, et, d'autre part, la difficulté ne commencet-elle pas pour les nombres fractionnaires eux-mêmes? " (Poincaré, La sScience et l'hypothèse, p. 50).

65. Cette définition implique en effet que soit donné l'ensemble des rationnels, puisqu'elle consiste à considérer différentes répartitions de ceux-ci. 
comporte le caractère nébuleux du continu physique, et c'est pourquoi toute mesure expérimentale est essentiellement approximative ou approchée $^{66}$. Par ailleurs, nous avons déjà insisté sur ce point, Poincaré affirme à de nombreuses reprises le caractère fondamentalement expérimental de la physique: si les lois physiques fournissent une véritable connaissance ${ }^{67}$, c'est seulement dans la mesure où elles sont vérifiées par des expériences. Cette vérification n'est autre que l'opération de mesure, qui repose sur l'institution d'un langage géométrique, c'est-à-dire sur le choix d'un groupe continu de déplacements. Ce caractère continu du groupe a ainsi pour vocation première d'assurer l'adéquation du langage de la mesure (c'està-dire de la géométrie) au donné brut de l'expérience. Or, c'est précisément cette adéquation que l'on ne comprend plus si le continu mathématique est conçu comme "logiquement discret ", ce qui est inévitable dès lors qu'il est défini à la façon "des plus récents géomètres». Et c'est pourquoi on ne comprend plus dès lors le succès de l'application des procédés du calcul infinitésimal à la connaissance de la nature. C'est en relation à ce point que nous revenons à présent sur les enseignements de la première partie du présent travail.

Nous avons vu que le caractère «logiquement discret» du continu ensembliste peut être compris en termes d'identité et de diversité, c'està-dire selon la logique du même et de l'autre. On est conduit, dans ce contexte, à interpréter le signe ' $=$ ' comme exprimant une identité, dans la mesure où une équation entre nombres rationnels ou réels de la forme 'a = $\mathrm{b}$ ' exprime que les signes $\mathrm{a}$ et $\mathrm{b}$ renvoient au même nombre, c'est-à-dire au même élément de l'ensemble des rationnels ou de l'ensemble des réels. Le caractère logiquement discret de ces ensembles implique en effet que deux éléments sont soit distincts soit numériquement identiques (au sens où l'on a affaire, soit à deux entités, soit à une même entité). Or, le physicien expérimental, ayant toujours affaire à des mesures qui sont intrinsèquement approximatives, ne peut interpréter ' $a=b$ ' en ces termes, car cette équation signifie nécessairement 'a est à peu près égal à b' ou 'b $-\mathrm{e}<\mathrm{a}$

66. Il est important de ne pas confondre cette thèse du caractère essentiellement approximatif de toute mesure expérimentale avec certains passages dans lesquels Poincaré écrit que l'on est souvent conduit, en physique, à assimiler un corps peu déformable à un solide géométrique idéal, qui obéit donc aux lois de la géométrie, et à laisser la physique prendre en charge seulement les déformations que l'on considérera par exemple comme des dilatations thermiques. Ce contexte n'est pas celui du rôle constitutif de la géométrie dans l'institution d'un système de mesure, mais celui de l'utilisation simplificatrice de considérations géométriques en physique. La différence est de taille et devient claire dès lors que l'on remarque que, pour que les déformations du corps puissent faire l'objet d'une étude physique, il faut qu'elles puissent être mesurées. A l'inverse, l'approximation essentielle de toute mesure physique ne saurait faire l'objet d'une mesure puisqu'elle appartient à l'instrument de mesure lui-même.

67. Contrairement aux principes qui ne sont ni vrais ni faux puisque ce sont des conventions. 
$<\mathrm{b}+\varepsilon^{\prime}$. Cela ne veut pas dire que ces dernières formules doivent être comprises, du point de vue mathématique, comme synonymes de ' $\mathrm{a}=\mathrm{b}$ ', mais cela interdit qu'elles aient une signification tout à fait différente, auquel cas on ne pourrait pas considérer que la mesure expérimentale donnant lieu à 'a est à peu près égal à b' constitue une vérification d'une équation de la forme ' $\mathrm{a}=\mathrm{b}$ ' (comprise cette fois-ci comme une exacte égalité). Ce serait pourtant le cas si l'on interprétait " =" comme le signe de l'identité numérique. En effet, le résultat d'une mesure doit toujours être donné sous la forme: ' $\mathrm{a}=\mathrm{b}+\varepsilon$ ', e étant non nul, inconnu et éventuellement très petit. Mais, quelle que soit la valeur (non nulle) de e, si l'on interprète '=' comme le signe de l'identité numérique, ' $a=b+\varepsilon$ ' a une signification fondamentalement différente de celle de ' $a=b$ ' puisqu'on est obligé d'effectuer un saut conceptuel pour passer de l'une à l'autre: on a en effet affaire à deux entités dans la première (elle implique que 'a' et 'b' renvoient à des éléments distincts) et à une seule dans la seconde (elle signifie que 'a' et 'b' renvoient au même élément), et on ne comprend plus alors en quoi la première pourrait constituer une vérification de la seconde. Pour le dire autrement, cela n'a pas de sens d'affirmer que deux choses sont plus ou moins identiques, plus ou moins la même ou à peu près identiques, à peu près la même si l'on entend par «identique» l'identité numérique. Selon la grammaire de celle-ci un signe renvoie en effet soit à la même chose qu'un autre signe, soit à une autre chose; l'identité numérique ne souffre pas l'approximation ${ }^{68}$. C'est ce qui la différencie de l'égalité mathématique ${ }^{69}$.

68. On pourrait objecter que l'altérité et la distinction des nombres rationnels et réels, revendiquée par le point de vue ensembliste, n'interdit pas que l'on puisse "mesurer" la distance qui sépare deux nombres et que le discours de l'approximation soit alors possible dans ce cadre. Nous répondrons que le même argument que celui que nous avons exposé, appliqué cette fois aux nombres mesurant cette distance, conduirait à conclure que ces nombres ne peuvent être soumis au régime de l'identité numérique. Et il serait alors problématique de persister à maintenir ce régime pour les premiers.

69. Notons que celle-ci ne saurait être caractérisée conceptuellement autrement que par ce trait qui la distingue de l'identité numérique. Si nous pouvions formuler en langage ordinaire «ce qu'est» l'égalité mathématique, celle-ci n'appartiendrait pas «à la seule langue que le physicien puisse parler». La généralisation de cette remarque explique l'ambiguité fréquente et le caractère le plus souvent négatif du discours philosophique de Poincaré.

Ajoutons que l'égalité, ici distinguée de l'identité numérique, ne correspond pas non plus à l'égalité entre choses, laquelle est définie comme la relation qui lie deux choses lorsqu'une propriété leur est commune. Par exemple, deux objets de même poids seront alors dits égaux (sous le rapport du poids). Une telle perspective ne saurait être celle de Poincaré, dans la mesure où elle s'inscrit dans une logique de la substance et de ses attributs, à laquelle le langage mathématique échappe précisément. Poincaré utilise néanmoins à l'occasion ce type de discours, ce qui est difficilement évitable puisque cette logique est inhérente au langage ordinaire.

Il en résulte que les analyses par Poincaré de la formule du continu physique ne sauraient être interprétées dans les termes du principe d'identité des indiscernables, même s'il s'agissait pour Poincaré d'établir que ce principe est faux dans le cadre de l'expérience mesurée. A la vérité, ce principe n'est pas ici faux, mais dénué de sens puisqu'il suppose le principe d'identité, dont nous venons de voir qu'il est lui aussi dénué de sens ici. 
C'est pourquoi, lorsque Poincaré emploie des expressions comme "approximativement identique ", il ne peut pas signifier l'identité numérique. Encore une fois, l'argument présenté ci-dessus est foncièrement négatif: il ne s'agit pas d'établir une caractérisation conceptuelle positive de l'égalité mathématique, mais de montrer qu'une interprétation du signe ' $=$ ' en des termes qui impliquent un saut conceptuel entre ' $=$ ' et ' $<$ ' ne peut être pertinente dans le cas de l'égalité de grandeurs mathématiques continues, car elle interdit de comprendre que ces grandeurs ont été faites "sur mesure» pour "y faire rentrer les faits sans dénaturer ce qu'ils ont d'essentiel ${ }^{70} »$. C'est parce que les relations d'égalité exacte entre les solides idéaux et entre les déplacements idéaux de la géométrie ne sont pas des relations d'identité qu'elles constituent un langage adéquat au donné sensible, où ne règne que l'approximation et donc l'inégalité.

L'interprétation ici défendue consiste ainsi à caractériser la "discrétion logique" par le saut conceptuel qui sépare l'identité et l'altérité et que l'on retrouve dans l' "abîme " qui sépare les substances. À l'inverse, l'égalité mathématique, si elle ne s'identifie pas à l'inégalité, n'en est pas conceptuellement distincte. C'est ce qui apparaît très clairement dans ce texte de Leibniz sur le principe de continuité:

Ainsi le mouvement, décroissant continuellement, se perd enfin dans le repos, l'inégalité continuellement diminuée se transforme en exacte égalité, de sorte que le repos peut être considéré comme un mouvement infiniment petit ou comme une lenteur infinie, et l'égalité comme une inégalité infiniment petite; et pour cette raison, tout ce qui a été démontré, soit du mouvement en général, soit de l'inégalité en général, doit aussi être vrai, selon notre conception, appliqué au repos ou à l'égalité, si bien que la règle du repos ou de l'égalité peut être conçue, en un certain sens, comme un cas spécial de la règle du mouvement ou de l'inégalité ${ }^{71}$.

Si l'on interprétait l'égalité mathématique comme une identité numérique, il serait légitime de remplacer ici «égalité » par «identité » et «inégalité » par «altérité». Mais le texte n'aurait alors manifestement aucun sens: Leibniz affirmerait que deux entités différentes se transformeraient de façon continue en une seule et même entité.

Notons ici que si Poincaré mentionne que la définition ensembliste du continu mathématique ne correspond pas à la notion du continu conçu comme unité dans la multiplicité, ce n'est pas directement cette inadéquation qui est philosophiquement insatisfaisante, mais l'impossibilité de comprendre que l'Analyse mathématique constitue l'outil central de la physique. L'argumentation développée ci-dessus ne s'appuie ainsi que sur ce dernier point et non sur l'inadéquation conceptuelle en question.

70. Poincaré, La Science et l'hypothèse, p. 26.

71. Leibniz, "Remarques sur la partie générale des principes de Descartes", p. 105, 107, 109. 
L'impossibilité de comprendre l'application du continu mathématique au donné de l'expérience (qui constitue un aspect de ce qui est visé par Poincaré lorsqu'il affirme que les mathématiques constituent le seul langage possible de la physique) dès lors que l'on interprète une égalité entre nombres rationnels ou réels comme l'identité de deux entités, permet donc de conclure au non-sens des considérations ontologiques relatives aux théories physiques et mathématiques: ne pouvant être comprises comme fonctionnant selon la logique de l'identité, elles ne sauraient en effet véhiculer une ontologie. Elle permet aussi de comprendre ce qui sous-tend le problème philosophique, posé par Poincaré, de l'adéquation des mathématiques du continu à la physique. Il reste maintenant à exposer la solution qu'il en donne.

\section{I.4. Infini actuel et infini potentiel}

L'une des raisons pour lesquelles Poincaré qualifie de "réalistes" les cantoriens est que ceux-ci, posant comme donnés les ensembles des réels et des rationnels, introduisent par là inévitablement la "discrétion logique » au sein des continus du premier et du second ordre, les soumettant ipso facto au régime de l'identité numérique, dont nous avons vu qu'elle caractérise le langage ontologique de la substance. Ceci entre en parfaite cohérence avec le fait que c'est en référence à la position de l'infini actuel que Poincaré parle de réalisme. En effet, c'est seulement en tant qu'ils sont considérés comme tous donnés en une collection que les nombres rationnels ou réels possèdent cette détermination d'altérité qui caractérise la discrétion logique ${ }^{72}$, autrement dit: que la «nébuleuse» du continu physique (caractérisée par l'indiscernabilité de termes contigus d'une série sensible) est "résolue en étoiles », pour reprendre une image de Poincaré. En revanche, au cours du processus indéfini consistant à résoudre la contradiction inhérente à la formule du continu physique par le remplacement de 'A = B' par ' $A<B$ ', ce caractère nébuleux du continu physique - dont nous avons vu qu'il est solidaire de l'indéfinie approximation des mesures physiques n'est pas perdu, puisque l'on sait que l'on sera toujours conduit à introduire un troisième terme $\mathrm{D}$ entre $\mathrm{A}$ et $\mathrm{B}$, tel que $\mathrm{A}=\mathrm{D}, \mathrm{D}=\mathrm{B}$, $\mathrm{A}<\mathrm{B}$ (formule du continu physique), c'est-à-dire tels que $\mathrm{A}$ et $\mathrm{D}$ (respectivement $\mathrm{D}$ et $\mathrm{B}$ ) sont indiscernables. Ainsi, la solution de Poincaré consiste à dire que si l'on considère l' «ensemble» des rationnels ${ }^{73}$ comme un infini potentiel (ce que signifie très exactement le processus décrit dans

72. S'il distingue le formaliste hilbertien de la logistique de Russell en affirmant que celui-là, contrairement à celle-ci, ne pose pas l'infini actuel, nous avons vu que Poincaré n'en insiste pas moins sur le fait que l'entreprise axiomatique de Hilbert implique que soient distingués des individus. C'est cette seule exigence qui nous intéresse ici.

73. La reconstruction génétique des nombres réels par Poincaré ne s'appuie pas sur la formule du continu physique mais est appelée par des intuitions de nature géométrique. C'est pourquoi nous avons concentré notre analyse sur celle des nombres rationnels. 
la phrase précédente) et non comme un infini actuel, l'idée qui préside à la résolution de la contradiction du continu physique est présente sans que le caractère nébuleux du continu physique soit perdu ${ }^{74}$. Un passage de l'article consacré à Cournot manifeste clairement le lien établi par Poincaré entre le refus du réalisme et la caractérisation du continu comme infini potentiel:

Ces infiniment petits, raisons des choses, sont-ils de perpétuels devenir comme les infiniment petits leibnitiens? Pour nous, qui ne croyons pas à la possibilité de concevoir un monde extérieur indépendamment du sujet pensant, ce serait la solution la plus simple et la plus naturelle. La raison première fuirait toujours devant l'esprit qui la cherche sans jamais pouvoir l'atteindre, et ce serait l'infiniment petit leibnitien qui symboliserait le mieux cette fuite éternelle ${ }^{75}$.

Nous ne saurions donc suivre Vuillemin lorsque celui-ci écrit:

L'infini est le genre dont le continu est une espèce: telle est la découverte fondamentale de Georg Cantor ${ }^{76}$.

Jusqu'à Georg Cantor, on n'a jamais clairement distingué le problème de l'infini et celui du continu. [...] Les philosophes anciens s'accordent à postuler que le continu ou infini n'existe pas en acte, mais seulement en puissance. D'Aristote à Poincaré, personne ou presque ne met ce postulat en question ${ }^{77}$.

$\mathrm{Si}$, en effet, Poincaré défend la thèse philosophique selon laquelle il faut comprendre le continu comme un infini potentiel, ce n'est pas faute de la questionner, mais parce qu'il trouve dans l'infini potentiel une vertu que ne possède pas l'infini actuel. Ce n'est donc pas seulement à cause des paradoxes qu'il engendre que Poincaré rejette l'infini actuel, c'est parce qu'en considérant l'infinité du continu comme actuelle et non pas comme potentielle, on ne voit pas en quoi il confère aux mathématique la spécificité qui en fait le langage privilégié de la physique. Car on s'interdit alors de prendre en compte et l'intuition sensible et l'intuition a priori qui président à la conception mathématique du continu et dont on ne comprend l'importance qu'une fois reconnue la vertu positive de l'infini potentiel. Telle semble être la vocation philosophique des reconstructions génétiques du continu et de la géométrie: tenter de mettre en évidence les instances sensibles et a priori qui y sont à l'œuvre, ainsi que leur articulation. Ainsi

74. Notons que, du point de vue de la constitution ontologique du discours du même et de l'autre, l'infini actuel et le fini se trouvent ainsi réunis sous le signe du «logiquement discret" et opposés à l'infinité potentielle du continu mathématique. Outre la singularité d'une telle répartition entre fini, infini potentiel et infini actuel, ceci montre bien que Poincaré ne réduit nullement l'infini potentiel au fini et que ce serait une erreur d'interprétation d'associer les vues de Poincaré à une conception finitiste des mathématiques.

75. Poincaré, L'opportunisme scientifique, p. 113.

76. Vuillemin, La philosophie de l'algèbre, p. 185.

77. Vuillemin, La philosophie de l'algèbre, p. 174-175. 
en est-il, en géométrie, de l'analyse du choix conventionnel d'un groupe de déplacements (décrit par Poincaré comme une catégorie de l'entendement) à l'occasion de l'expérience de la compensation au sein du continu physique. Nous avons vu que cette analyse suppose celle du continu mathématique, dont la genèse est rendue possible par une intuition a priori qui est la même que celle qui confère au principe du raisonnement par récurrence son évidence et son statut de jugement synthétique a priori:

Pourquoi donc ce jugement s'impose-t-il à nous avec une irrésistible évidence? C'est qu'il n'est que l'affirmation de la puissance de l'esprit qui se sait capable de concevoir la répétition indéfinie d'un même acte dès que cet acte est une fois possible. L'esprit a de cette puissance une intuition directe et l'expérience ne peut être pour lui qu'une occasion de s'en servir et par là d'en prendre conscience ${ }^{78}$.

Ce n'est pas seulement pour échapper à cette contradiction contenue dans les données empiriques que l'esprit est amené à créer le concept d'un continu, formé d'un nombre indéfini de termes.

Tout se passe comme pour la suite des nombres entiers. [...]

Dès que nous avons été amenés à intercaler des moyens entre deux termes consécutifs d'une série, nous sentons que cette opération peut être poursuivie au-delà de toute limite et qu'il n'y a pour ainsi dire aucune raison intrinsèque de s'arrêter ${ }^{79}$."

\section{Comme l'écrit G. Heinzmann:}

According to Poincaré, this constitution of sets [defined by the axiom of comprehension] is based on a methodological inversion which already concerns the philosophical presuppositions, i. e. the ontological hypotheses implied by the choice of a language. The cause of the antinomies resides, according to Poincaré, in an implicit recourse to a «false » intuition regarding abstract entities; in fact, for an anti-platonist, which was Poincaré's case from 1906 on, conceptual realism makes a usurping use of intuition when it relates it to the evident mode of presentation of an abstract entity instead of relating it to the capability to follow an action-schema ${ }^{80}$.

78. Poincaré, La Science et l'hypothèse, p. 41.

79. Poincaré, La Science et l'hypothèse, p. 53. La formule choisie par Poincaré: "répétition indéfinie d'un même acte dès que cet acte est une fois possible» donne lieu à l'objection suivante: l'usage du mot "même " n'implique-t-il pas que l'identité se retrouve au cœur même de ce qui constitue l'une des instances a priori fondamentales de la pensée mathématique pour Poincaré? Il faut répondre qu'ici encore, on ne peut comprendre cet usage comme faisant référence à l'identité numérique. En effet, un texte de La Science et l'hypothèse montre que cette puissance de l'esprit doit pouvoir se prêter au discours physique de l'approximation, comme cela est explicitement énoncé dans la dernière phrase:

"On peut se demander pourquoi, dans les sciences physiques, la généralisation prend volontiers la forme mathématique. La raison est maintenant facile à voir; ce n'est pas seulement parce que l'on a à exprimer des lois numériques; c'est parce que le phénomène observable est dû à la superposition d'un grand nombre de phénomènes élémentaires tous semblables entre eux; ainsi s'introduisent tout naturellement les équations différentielles. 
Si les définitions rigoureuses du continu mathématique sont philosophiquement insatisfaisantes, c'est précisément parce que leur vocation est de ne pas faire appel à l'intuition, laquelle, nous venons de le voir, joue un rôle central dans la réponse à la question du rôle du continu mathématique au sein de la connaissance physique. C'est de même parce qu'elles interdisent de comprendre ce que permettent les intuitions (sensibles et a priori) qui gouvernent la conception mathématique du continu que les considérations ontologiques sont dénuées de sens lorsqu'elles portent sur les théories physiques. Pour terminer, citons deux textes extraits de Science et méthode qui suggèrent que, selon Poincaré, s'interdire de répondre à cette question en ne prenant en considération que les définitions "logiques", c'est aussi s'interdire de comprendre ce qui constitue "la réalité véritable » des mathématiques elles-mêmes; et c'est donc manquer le but de la philosophie des sciences telle que la conçoit Poincaré.

«Prenons par exemple l'idée de fonction continue. Ce n'est d'abord qu'une image sensible, un trait tracé à la craie sur le tableau noir. Peu à peu elle s'épure; on s'en sert pour construire un système compliqué d'inégalités, qui reproduit toutes les lignes de l'image; quand tout a été terminé, on a décintré, comme après la construction d'une voûte; cette représentation grossière, appui désormais inutile, a disparu, et n'est resté que l'édifice luimême, irréprochable aux yeux du logicien. Et pourtant, si le professeur ne rappelait l'image primitive, s'il ne rétablissait momentanément le cintre, comment l'élève devinerait-il par quel caprice toutes ces inégalités se sont échafaudées de cette façon les unes sur les autres? La définition serait logiquement correcte, mais elle ne lui montrerait pas la réalité véritable ${ }^{81}$.»

[...] les mathématiques nous apprennent, en effet, à combiner le semblable au semblable. Leur but est de deviner le résultat d'une combinaison, sans avoir besoin de refaire cette combinaison pièce à pièce. Si l'on a à répéter plusieurs fois une même opération, elles nous permettent d'éviter cette répétition en nous en faisant connaître d'avance le résultat par une sorte d'induction. Je l'ai expliqué plus haut, dans le chapitre sur le raisonnement mathématique.

Mais, pour cela, il faut que toutes ces opérations soient semblables entre elles; dans le cas contraire, il faudrait évidemment se résigner à les faire effectivement l'une après l'autre et les mathématiques deviendraient inutiles.

C'est donc grâce à l'homogénéité approchée de la matière étudiée par les physiciens que la physique mathématique a pu naître." (Poincaré, La Science et l'hypothèse, p. 171, 172)

Un argument analogue à celui exposé plus haut conduirait à montrer que cette application du principe à un donné où règne l'approximation implique que l'«identité " qui apparaît dans sa formulation doit être comprise comme une égalité.

Ce texte s'inscrit explicitement dans la problématique de la nature mathématique de la physique. Il concerne plus particulièrement l'élaboration des lois physiques. Ayant choisi de concentrer notre étude sur la mesure physique, nous n'avons pas traité de cet autre aspect fondamental de la problématique de l'application du calcul différentiel et intégral à la connaissance physique.

80. Heinzmann, «Poincaré on understanding mathematics», p. 147.

81. Poincaré, Science et méthode, p. 111. 
«Parmi toutes les constructions que l'on peut combiner avec les matériaux fournis par la logique, il faut faire un choix; le vrai géomètre fait ce choix judicieusement parce qu'il est guidé par un sûr instinct, ou par quelque vague conscience de je ne sais quelle géométrie plus profonde, et plus cachée, qui seule fait le prix de l'édifice construit.

Chercher l'origine de cet instinct, étudier les lois de cette géométrie profonde qui se sentent et ne s'énoncent pas, ce serait encore une belle tâche pour les philosophes qui ne veulent pas que la logique soit tout ${ }^{82}$.»

\section{Bibliographie}

Aristote, Catégories, traduit par F. Ildefonse et J. Lallot, Paris, Editions du Seuil, 2002.

Benacerraf, Paul, «What numbers could not be ", dans Putnam, H., Benacerraf, P., dir., Philosophy of mathematics. Selected readings, New York, Cambridge University Press, 1996, p. 272-294.

Bouveresse, Jacques, Le pays des possibles, Paris, Les Editions de Minuit, 1988.

Brunschvicg, Léon, Les étapes de la philosophie mathématique, Paris, Blanchard, 1993.

Descartes, René, Principes de la philosophie, Paris, Vrin, 1990.

Folina, Janet, "Logic and intuition in Poincaré's philosophy of mathematics », dans Greffe, J.L., Heinzmann, G., Lorenz, K., dir., Henri Poincaré. Science et philosophie, Berlin/Paris, Akademie Verlag/Blanchard, 1996, p. 417-434

Frege, Gottlob, Ecrits logiques et philosophiques, traduit par C. Imbert, Paris, Editions du Seuil, 1970.

Galilée, Il Saggiatore, traduit par C. Chauviré, Annales littéraires de l'Université de Besançon, Les Belles Lettres, 1979.

Galilée, Dialogue sur les deux grands systèmes du monde, traduit par René Fréreux, Paris, Editions du Seuil, 1992.

Giedymin, Jerzy, Science and convention, Oxford, Pergamon Press, 1982.

Greffe, J.L., Heinzmann, G., Lorenz, K., dir., Henri Poincaré. Science et philosophie, Berlin/Paris, Akademie Verlag/Blanchard, 1996

Heinzmann, Gerhard, "Poincaré on understanding mathematics ", Philosophia Scientiae, 3.2, 1998/99, p. 143-160.

Hilbert, David, Les fondements de la géométrie, traduit par P. Rossier, Paris, Dunod, 1971.

Husserl, Edmund, Philosophie de l'arithmétique, traduit par J. English, Paris, Presses Universitaires de France, 1992.

Jacob, Pierre, dir., De Vienne à Cambridge, Paris, Gallimard, 1980.

Kant, Emmanuel, Critique de la raison pure, Paris, Gallimard, Bibliothèque de la Pléiade, 1986.

Leibniz, "Remarques sur la partie générale des principes de Descartes", dans Leibniz, Opuscules philosophiques choisis, traduit par P. Schrecker, Paris, Vrin, 2001, p. 31-159.

Poincaré, Henri, La Science et l'hypothèse, Paris, Flammarion, 1968.

Poincaré, Henri, La Valeur de la science, Paris, Flammarion, 1970. 
Poincaré, Henri, Science et Méthode, Paris, Kimé, 1999.

Poincaré, Henri, Dernières pensées, Paris, Flammarion, 1930.

Poincaré, Henri, Des fondements de la géométrie, Paris, Chiron, 1921.

Poincaré, Henri, L'opportunisme scientifique, Basel, Boston, Berlin, Birkhäuser, 2002.

Putnam, Hilary, Philosophie de la logique, traduit par P. Peccatte, Combas, Editions de l'éclat, 1996.

Putnam, H., Benacerraf, P., dir., Philosophy of mathematics. Selected readings, New York, Cambridge University Press, 1996.

Quine, Willard Van Orman, From a logical point of view, Cambridge, Harvard University Press, 1994.

Quine, Willard Van Orman, Le mot et la chose, traduit par J. Dopp et P. Gochet, Paris, Flammarion, 1977.

Quine, Willard Van Orman, "Parler d'objets », dans Quine, Willard Van Orman, Relativité de l'ontologie et autres essais, traduit par J. Largeault, Paris, Aubier, 1977, p. 13-37.

Quine, Willard Van Orman, "Le domaine et le langage de la science ", dans Jacob, Pierre, dir., De Vienne à Cambridge, Paris, Gallimard, 1980, p. 219-240.

Vuillemin, Jules, La philosophie de l'algèbre, Paris, Presses Universitaires de France, 1962.

Wittgenstein, Ludwig, Remarks on the foundations of mathematics, traduit par G. E. M. Anscombe, Oxford, Blackwell, 1994.

Zahar, Elie, Poincarés philosophy, Chicago, Open Court, 2001. 